Max-Planck-Institut für demografische Forschung

Max Planck Institute for Demographic Research

Konrad-Zuse-Strasse 1 - D-18057 Rostock - GERMANY

Tel +49 (0) 3812081 - 0; Fax +49 (0) 3812081 - 202;

http://www.demogr.mpg.de

MPIDR TECHNICAL REPORT 2013-001

AUGUST 2013

\title{
Comparison of DemoDiff Releases \\ 2.0 and 3.0
}

Rainer Walke (walke@demogr.mpg.de)

For additional material see www.demogr.mpg.de/tr/

This technical report has been approved for release by: Dirk Vieregg (vieregg@demogr.mpg.de),

Coordinator of the IT Group.

(C) Copyright is held by the authors.

Technical reports of the Max Planck Institute for Demographic Research receive only limited review. Views or opinions expressed in technical reports are attributable to the authors and do not necessarily reflect those of the Institute. 


\title{
Comparison of DemoDiff Releases 2.0 and 3.0
}

\author{
Rainer Walke, MPIDF Rostock*
}

2013-Aug-26

\begin{abstract}
In this Technical Report, results of the program compareFinRaw are presented. compareFinRaw is a tool that is particularly useful for comparing large data sets. It is typically used to compare different releases of the same data. In this example, we compare two different releases of the project DemoDiff. To be more precise, we compare the five revised files of DemoDiff Release 3.0 with the older Release 2.0 files. It should provide users of the DemoDiff data a comprehensive overview on all manipulations of the data that have occurred from Release 2.0 to Release 3.0.
\end{abstract}

Keywords data analysis, data comparability, data evaluation, data processing, software

\section{Introduction}

In a Technical Report by Walke and Müller [TR-2012-003] we have described a procedure to compare two datasets with little conditions. Only the ID had to be the same in each row of both datasets. The program that compares the datasets had been named compareFinRaw. It compares each column (variable) from one set with each column from the second data set. It furthermore checks whether there are bijective mappings between variables of the two data sets. If there is no direct mapping it computes how much the levels of the variables have to be changed to get a bijective mapping.

In this report, we use compareFinRaw to compare two different releases of the project DemoDiff. We are comparing Release 2.0 [DemoDiff 2.0] and Release 3.0 [DemoDiff 3.0]. Both are available by GESIS (www.gesis.org). We compare the following data sets: anchor1_DD.dta, anchor2_DD.dta, partner1_DD.dta, partner2_DD.dta and weights.dta ${ }^{1}$.

We are using the statistical package $\mathrm{R}$ [R 2.15] for all the computations and RStudio, knitR and MiKTeX for the documentation. The output of these programs is a readable list that reports the number and the names of variables that completely match between the two releases, i.e. have the same name and same content. The program also identifies all modified variables such as renamed variables, bijective recoded variables and variables with marked differences. Furthermore we get the names of all variables without variation at all. We hope, that this material helps users of DemoDiff to get a clear picture on what has been changed between the two releases. It should be noted that, depending on the number of variables compareFinRaw takes minutes or hours to compare the files. ${ }^{2}$.

\footnotetext{
*MPIDF, Konrad-Zuse-Straße 1, D-18057 Rostock, Germany. E-Mail: walke@demogr.mpg.de

${ }^{1}$ There are further files in the releases. However, they have been omitted because they are not suitable for comparison.

${ }^{2}$ In our case, it took 7 hours to run the program for the largest data set (anchor2_DD.dta)
} 


\section{Classification}

The idea is to classify all variables in data set $\mathbf{A}$ in respect to their relations to variables from data set $\mathbf{B}$.

A typical solution would be first to check whether a variable with the same name is available in data set $\mathbf{B}$. If this is true we could distinguish between identical and non identical content. This way we would classify all variable from set $\mathbf{A}$ into one of three possible categories.

This would give only a rough picture of the differences between the data sets. To detect renamed and recoded variables as well we use a more sophisticated classification scheme.

\section{classification tree}

1. The selected variable from $\mathbf{A}$ does not have variation.

$1.1[\mathrm{x}]$ No variable with the same name is available in data set $\mathbf{B}$.

1.2 One variable with the same name is available in data set $\mathbf{B}$.

$1.21[\mathrm{n}]$ Both variables are not identical.

1.22 [ni] Both variables are identical.

2. The selected variable from $\mathbf{A}$ does have variation.

2.1 There exists no bivariate mapping to one of the variables in data set $\mathbf{B}$.

$2.11[\mathrm{v}]$ No variable with the same name is available in data set $\mathbf{B}$.

2.12 [vn] One variable with the same name is available in data set $\mathbf{B}$.

2.2 There exists at least one bivariate mapping to one of the variables in data set $\mathbf{B}$.

2.21 There are more than one bivariate mappings.

2.211 All mapped variables from $\mathbf{B}$ do have a different name.

2.2111 [vb] No mapped variable from B is identical.

2.2112 [vbi] At least one mapped variable from $\mathbf{B}$ is identical.

2.212 One mapped variable from $\mathbf{B}$ has the same name.

2.2121 [vbn] The mapped variable from $\mathbf{B}$ is not identical.

2.2122 [vbni] The mapped variable from $\mathbf{B}$ is identical.

2.22 There exists exactly one bivariate mapping.

2.221 The mapped variable from $\mathbf{B}$ has a different name.

2.2211 [vb1] Both variables are not identical.

2.2212 [vb1i] Both variables are identical.

2.222 The mapped variable from $\mathbf{B}$ has the same name.

2.2221 [vb1n] Both variables are not identical.

2.2222 [vb1ni] Both variables are identical.

Every variable out of $\mathbf{A}$ will be assign to exactly one of these 13 classes. 


\section{Results and Summary}

The appendix contains the documented code. For each of the five data sets the code follows

the same logic.

1. Analyze all variables without variation

2. Analyze all variables with variation, but without a bijective mapping

3. Analyze all variables with variation and with a bijective mapping

4. Provide a comparison summary

As an example we display here the summary output that our program has produced for the data set anchor2_DD.dta.

\begin{tabular}{rrr} 
class & Release 2 & Release 3 \\
\hline $\mathrm{x}$ & 8 & 0 \\
$\mathrm{n}$ & 15 & 37 \\
$\mathrm{ni}$ & 612 & 612 \\
\hline $\mathrm{v}$ & 1 & 7 \\
$\mathrm{vn}$ & 369 & 359 \\
\hline $\mathrm{vb}$ & 2 & 0 \\
$\mathrm{vbi}$ & 11 & 1 \\
vbn & 2 & 1 \\
vbni & 954 & 956 \\
vb1 & 4 & 5 \\
vb1i & 3 & 2 \\
vb1n & 3 & 4 \\
vb1ni & 1262 & 1260 \\
\hline sum & 3246 & 3244
\end{tabular}

The results may be interpreted as follows:

Most variables are unchanged (ni, vbni, vb1ni). 612 variables do not have variation and are identical. $954+$ 1262 variables (vbni, vb1ni, Release 2) are unchanged, but have variation. There is a weak probability that 954 variables (vbni) show some redundancy.

8 constant variables (x, Release 2) have been dropped. One variable (v, Release 2) with variation has been dropped as well.

15 variables (n, Release 2) have been gained variation or have changed to another constant value.

2 variables (vb, Release 2 ) have been (less probably) renamed and recoded. 11 variables (vbi, Release 2) have been (less probably) renamed. 2 variables (vbn, Release 2) have been (probably) recoded.

4 variables (vb1, Release 2) have been (probably) renamed and recoded. 3 variables (vb1i, Release 2) have been (probably) renamed. 3 variables (vb1n, Release 2) have been (probably) recoded.

369 variables (vn, Release 2) had been changed between releases. They have the same name, but there exist no bijective mapping. The provided Levenshtein distance (appendix) gives a rough idea whether only some cases have been changed or not.

\section{Acknowledgements}

The report was supported by the Max Planck Institute for Demographic Research, Rostock, Germany. The author would like to thank Michaela Kreyenfeld, Rüdiger Lenke and Tom Hensel for valuable comments on the paper. I thank Andreas Müller for helping with the compareFinRaw.r code preparation. 


\section{A. anchor1}

Here we compare the data set anchor1_dd.dta from DemoDiff Release 2.0 with the same data set from DemoDiff Release 3.0.

We have to load the produced data collection. Release 2.0 files are denoted with $\mathbf{R}$ and Raw, release 3.0 files with $\mathbf{F}$ and Fin, respectively.

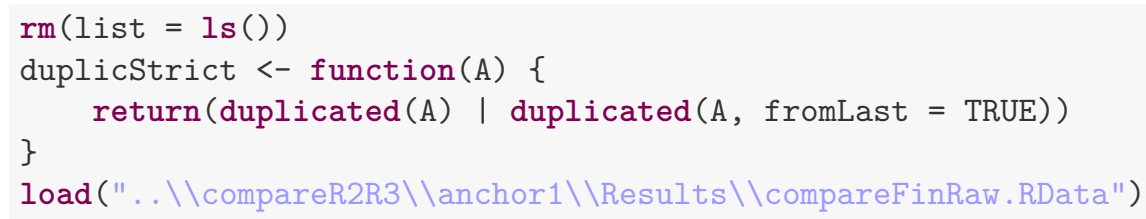

\section{A.1. no variation}

We compare the variables without variation first.

$($ R.x $<-\operatorname{sum}($ ! (RawNames $[R n v] \%$ in\% FinNames $))$ )

\#\# [1] 1

$($ F.x $<-$ sum(! (FinNames $[$ Fnv] $\%$ in\% RawNames $))$ )

\#\# [1] 0

\# added or lost variance

(R.nA <- sum (! (RawNames [Rnv] \%in\% FinNames [Fnv])) - R.x)

\#\# [1] 0

$(\mathrm{F} . \mathrm{nA}<-\operatorname{sum}($ ! (FinNames $[\mathrm{Fnv}] \%$ in\% RawNames $[\mathrm{Rnv}]))-\mathrm{F} . \mathrm{x})$

\#\# [1] 0

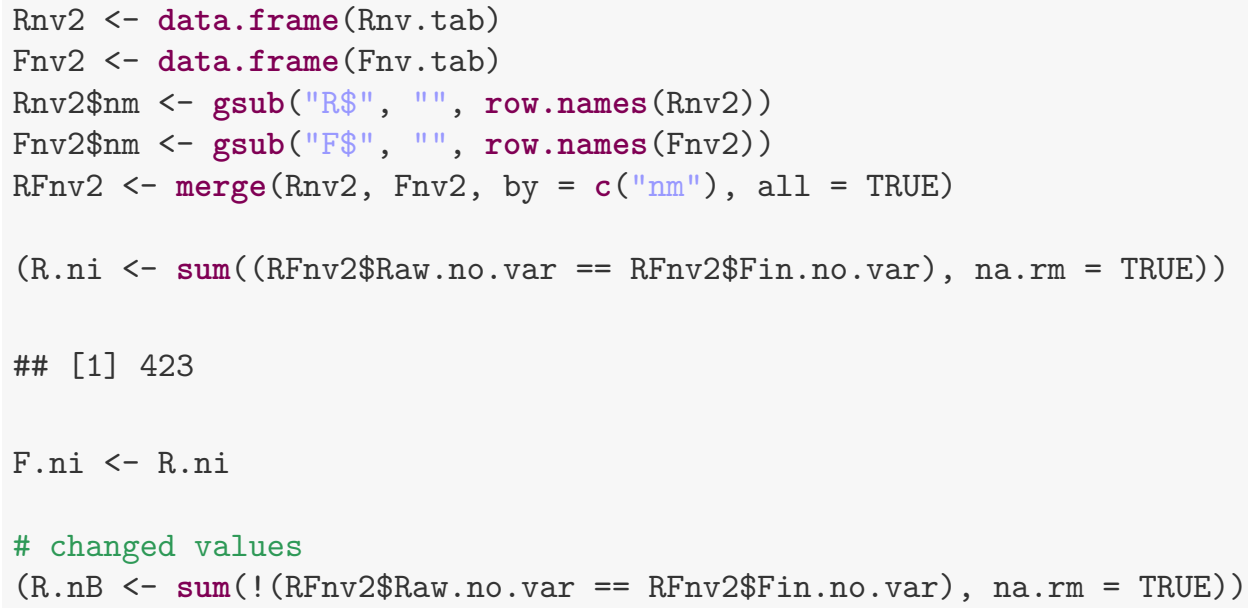




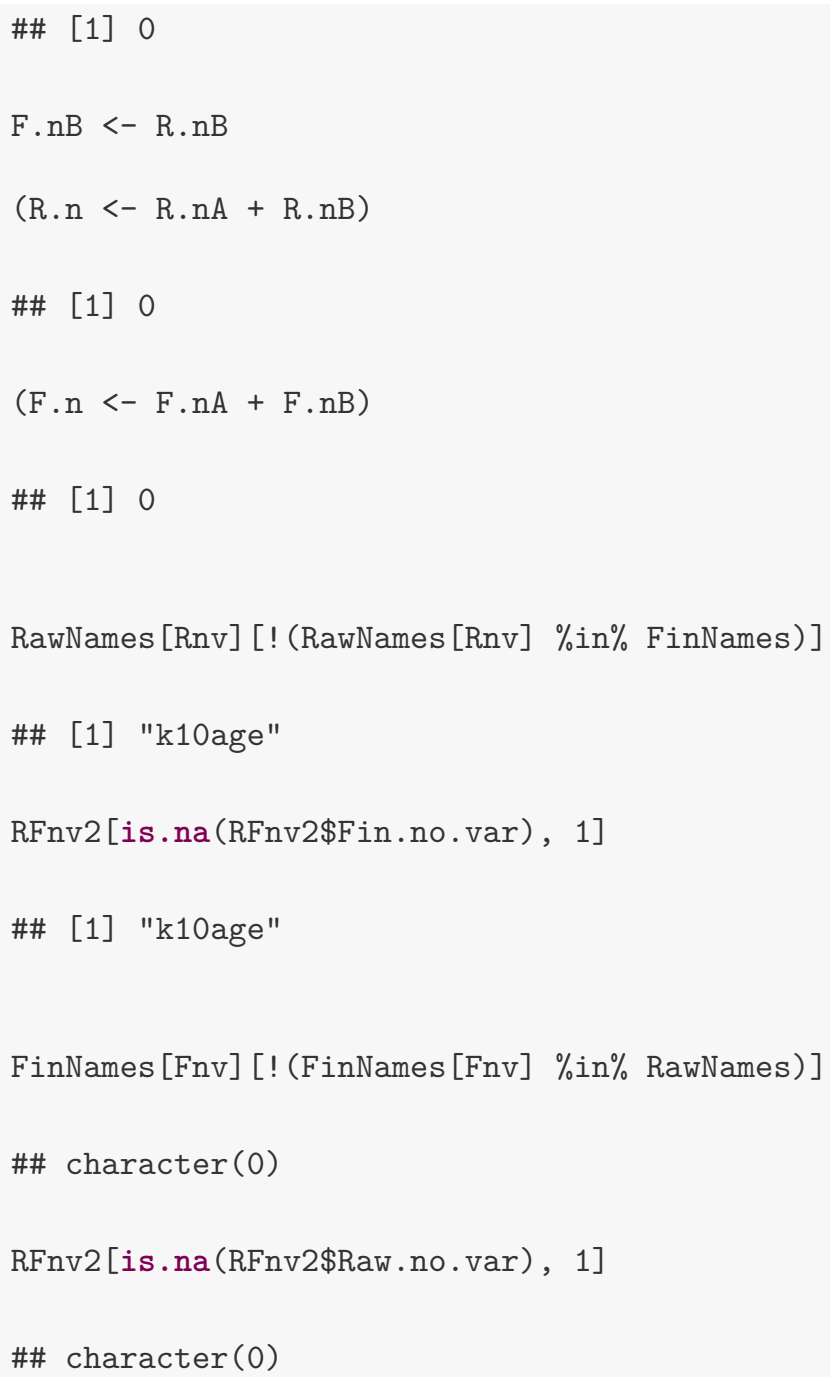

Release 2.0 For 1 variables without variation (k10age) exist no variables with the same name in the comparison data set. 423 variables without variation are identical in both data sets.

Release 3.0 423 variables without variation are identical in both data sets.

\section{A.2. variation, but no bijective mapping}

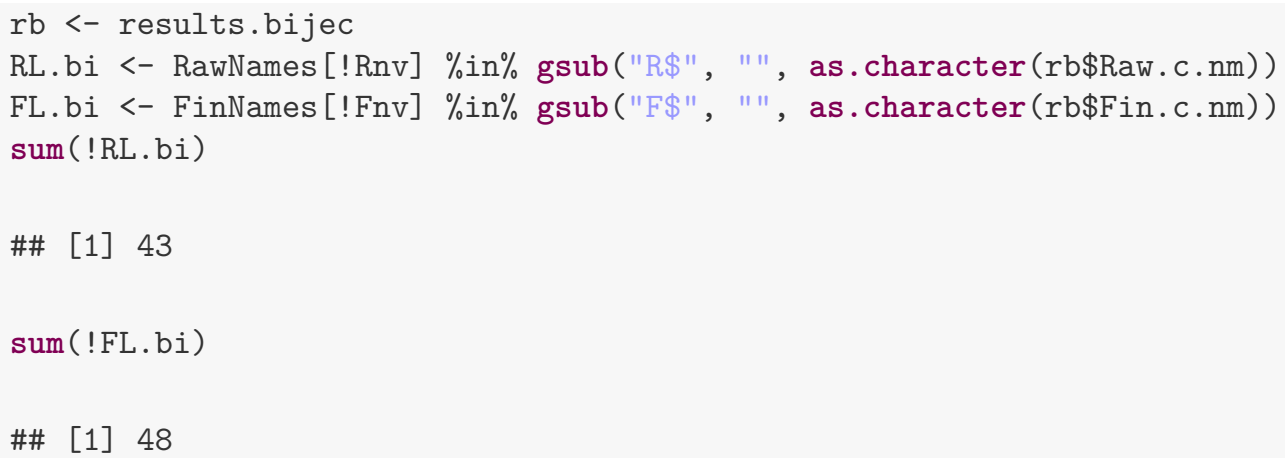


(R.v <- sum(! (RawNames [!Rnv] [!RL.bi] \%in\% FinNames))

\#\# [1] 0

RawNames [!Rnv] [!RL.bi] [! (RawNames [!Rnv] [!RL.bi] \%in\% FinNames)]

\#\# character $(0)$

(R.vn $<-$ sum(RawNames [!Rnv] [!RL.bi] \%in\% FinNames))

\#\# [1] 43

RawNames [!Rnv] [!RL.bi] [RawNames [!Rnv] [!RL.bi] \%in\% FinNames]

\begin{tabular}{|c|c|c|c|c|c|}
\hline [1] & "sex" & "dobm" & "sd4g" & "sdp1m" & "sdp1y" \\
\hline [6] & "frt11v1i1" & "frt11v1i2" & "frt11v1i3" & "frt11v1i4" & "frt11v1i5" \\
\hline [11] & "frt11v1i6" & "frt11v1i7" & "frt11v1i8" & "frt11v2i1" & 't11v2i2' \\
\hline [16] & "frt11v2i3" & "frt11v2i4" & "frt11v2i5" & "frt11v2i6" & Ert11v2i7' \\
\hline [21] & "frt11v2i8" & "flag1" & "flag18" & "sex_gen" & ex_gen" \\
\hline [26] & "dobm_gen" & "pdoby_gen" & "pdobm_gen" & "age" & "page" \\
\hline [31] & "mage" & "fage" & "k1age" & "k2age" & "homosex" \\
\hline [36] & "infertile" & "pregnant" & "hhsizemrd" & "othmrd" & "hhcomp" \\
\hline [41] & "isei" & "siops" & "hhincgcee" & & \\
\hline
\end{tabular}

$($ F.v $<-\operatorname{sum}(!($ FinNames [!Fnv] [!FL.bi] \%in\% RawNames $))$ )

\#\# [1] 5

FinNames [!Fnv] [!FL.bi] [! (FinNames [!Fnv] [!FL.bi] \%in\% RawNames)]
\#\# [1] "ykage"
\#\# [4] "kldb2010"
"ykid"
"isco08"
"flag_isco08_kldb2010"
(F.vn $<-$ sum(FinNames [!Fnv] [!FL.bi] $\%$ in \% RawNames)
\#\# [1] 43

FinNames [!Fnv] [!FL.bi] [FinNames [!Fnv] [!FL.bi] \%in\% RawNames]
\# [1] "sex" "dobm" "sd4g" "sdp1m" "sdp1y"
\#\# [6] "frt11v1i1" "frt11v1i2" "frt11v1i3" "frt11v1i4" "frt11v1i5"
\#\# [11] "frt11v1i6" "frt11v1i7" "frt11v1i8" "frt11v2i1" "frt11v2i2"
\#\# [16] "frt11v2i3" "frt11v2i4" "frt11v2i5" "frt11v2i6" "frt11v2i7"
\#\# [21] "frt11v2i8" "flag1" "flag18" "sex_gen" "psex_gen"
\#\# [26] "dobm_gen" "pdoby_gen" "pdobm_gen" "age" "page"
\#\# [31] "mage" "fage" "k1age" "k2age" "homosex"
\#\# [36] "infertile" "pregnant" "hhsizemrd" "othmrd" "hhcomp"
\#\# [41] "isei" "siops" "hhincgcee" 
Release 2.0 43 variables share the name with one of the variables in Release 3.0 at least.

Release 3.0 For 5 variables with variation but without any bijective mapping exist no variables with the same name in the Release 2.0 data. 43 variables share the name with one of the variables in Release 2.0 at least.

We compare all variables pairs (with variation) which share the same name but are not connected with a bijective mapping. Those variables imply different information. DemoDiff data user should check whether they are using those variables for possible effects on their research. (The number of pairs may differ from 43 and 43).

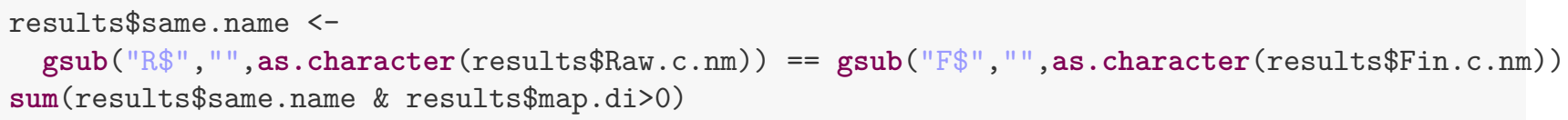

\begin{tabular}{|c|c|c|c|c|c|c|}
\hline \#\# & Fin.c.nm & Fin.c.ls & Raw.c.nm & Raw.c.ls & map.di & ed.di.sum \\
\hline \#\# & sexF & 2 & sexR & 2 & 4 & 2 \\
\hline \#\# & dobmF & 12 & dobmR & 12 & 10 & 7 \\
\hline \#\# & $s d 4 g F$ & 3 & sd4gR & 3 & 4 & 4 \\
\hline \#\# & $\mathrm{sdp} 1 \mathrm{mF}$ & 17 & $\mathrm{sdp} 1 \mathrm{mR}$ & 17 & 20 & 17 \\
\hline \#\# & sdp1yF & 45 & sdp1yR & 45 & 30 & 25 \\
\hline \#\# & frt11v1i1F & 10 & frt11v1i1R & 9 & 1 & \\
\hline \#\# & frt11v1i2F & 10 & frt11v1i2R & 9 & 1 & 1 \\
\hline \#\# & frt11v1i3F & 10 & frt11v1i3R & 9 & 1 & \\
\hline \#\# & frt11v1i4F & 10 & frt11v1i4R & 9 & 1 & \\
\hline \#\# & frt11v1i5F & 10 & frt11v1i5R & 9 & 1 & \\
\hline \#\# & frt11v1i6F & 10 & frt11v1i6R & 9 & 1 & \\
\hline \#\# & frt11v1i7F & 9 & frt11v1i7R & 8 & 1 & \\
\hline \#\# & frt11v1i8F & 10 & frt11v1i8R & 9 & 1 & \\
\hline \#\# & frt11v2i1F & 6 & frt11v2i1R & 5 & 1 & \\
\hline \#\# & frt11v2i2F & 6 & frt11v2i2R & 5 & 1 & \\
\hline \#\# & frt11v2i3F & 6 & frt11v2i3R & 5 & 1 & \\
\hline \#\# & frt11v2i4F & 6 & frt $11 \mathrm{v} 2 i 4 \mathrm{R}$ & 5 & 1 & \\
\hline \#\# & frt11v2i5F & 6 & frt11v2i5R & 5 & 1 & \\
\hline \#\# & frt11v2i6F & 6 & frt11v2i6R & 5 & 1 & \\
\hline \#\# & frt11v2i7F & 6 & frt11v2i7R & 5 & 1 & \\
\hline \#\# & frt11v2i8F & 6 & frt11v2i8R & 5 & 1 & \\
\hline \#\# & $\mathrm{flag} 1 \mathrm{~F}$ & 2 & flag1R & 2 & 4 & \\
\hline \#\# & $\mathrm{flag} 18 \mathrm{~F}$ & 3 & flag18R & 3 & 2 & \\
\hline \#\# & sex_genF & 2 & sex_genR & 2 & 4 & \\
\hline \#\# & psex_genF & 3 & psex_genR & 3 & 4 & \\
\hline \#\# & dobm_genF & 12 & dobm_genR & 12 & 10 & 7 \\
\hline \#\# & pdoby_genF & 45 & pdoby_genR & 45 & 30 & 25 \\
\hline \#\# & pdobm_genF & 17 & pdobm_genR & 17 & 20 & 17 \\
\hline \#\# & ageF & 9 & ageR & 9 & 2 & \\
\hline \#\# & pageF & 45 & pageR & 45 & 36 & 25 \\
\hline \#\# & mageF & 43 & mage R & 43 & 16 & 13 \\
\hline \#\# & fageF & 46 & fageR & 46 & 20 & 12 \\
\hline \#\# & k1ageF & 29 & k1ageR & 29 & 4 & \\
\hline \#\# & k2ageF & 26 & k2ageR & 26 & 4 & \\
\hline \#\# & homosexF & 3 & homosexR & 3 & 4 & \\
\hline
\end{tabular}




$\begin{array}{rr}\# \# & \text { infertileF } \\ \# \# & \text { pregnantF } \\ \# \# & \text { hhsizemrdF } \\ \# \# & \text { othmrdF } \\ \# \# & \text { hhcompF } \\ \# \# & \text { iseiF } \\ \# \# & \text { siopsF } \\ \# \# & \text { hhincgceeF }\end{array}$

$\begin{array}{rr}3 & \text { infertileR } \\ 4 & \text { pregnantR } \\ 9 & \text { hhsizemrdR } \\ 6 & \text { othmrdR } \\ 15 & \text { hhcompR } \\ 174 & \text { iseiR } \\ 177 & \text { siopsR } \\ 330 & \text { hhincgceeR }\end{array}$

3
4
9
6
15
60
57
331

$\begin{array}{rr}2 & 2 \\ 4 & 6 \\ 2 & 1 \\ 2 & 1 \\ 2 & 1 \\ 462 & 2486 \\ 478 & 2529 \\ 1 & 7\end{array}$

\section{A.3. variation and bijective mapping}

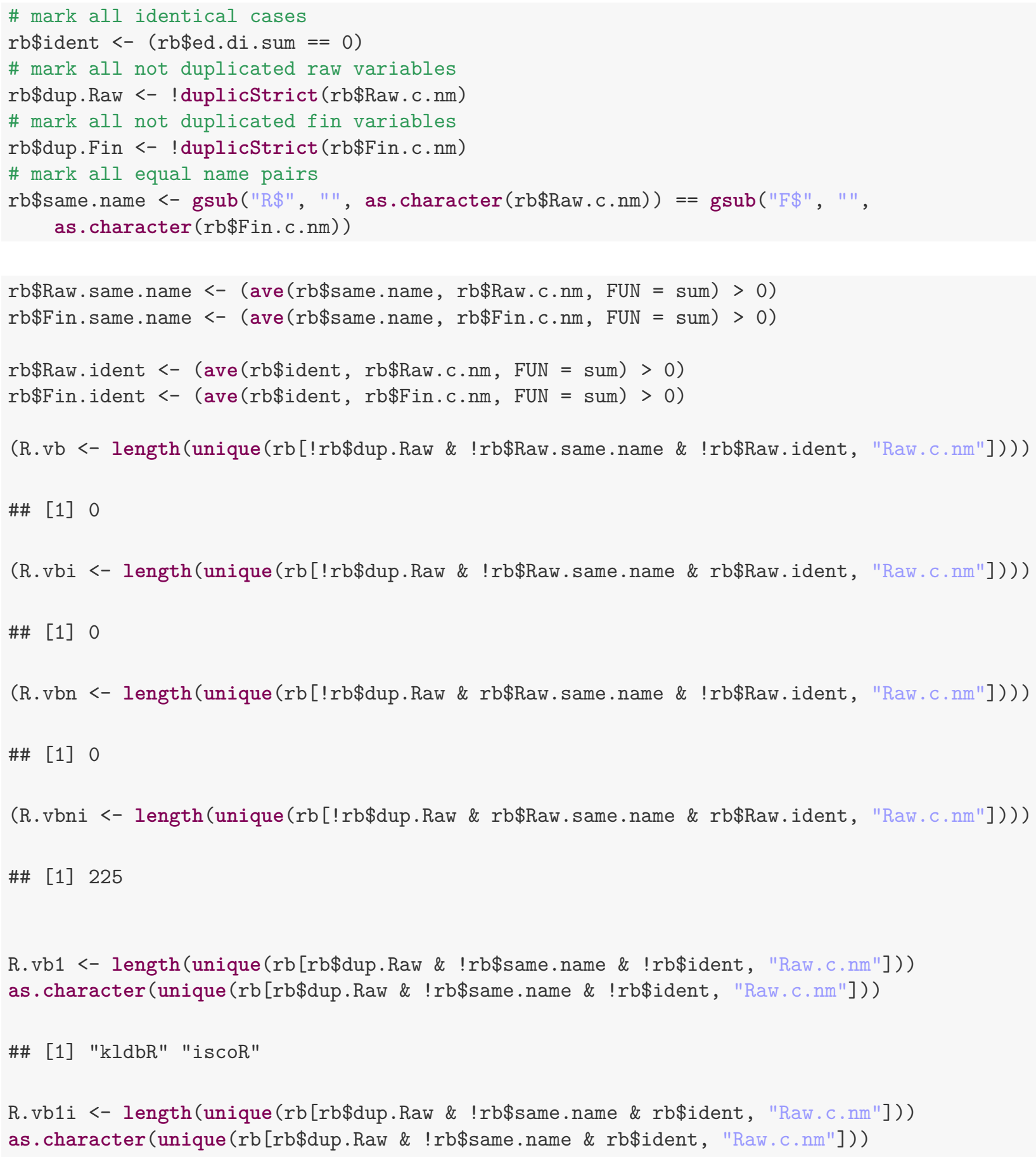




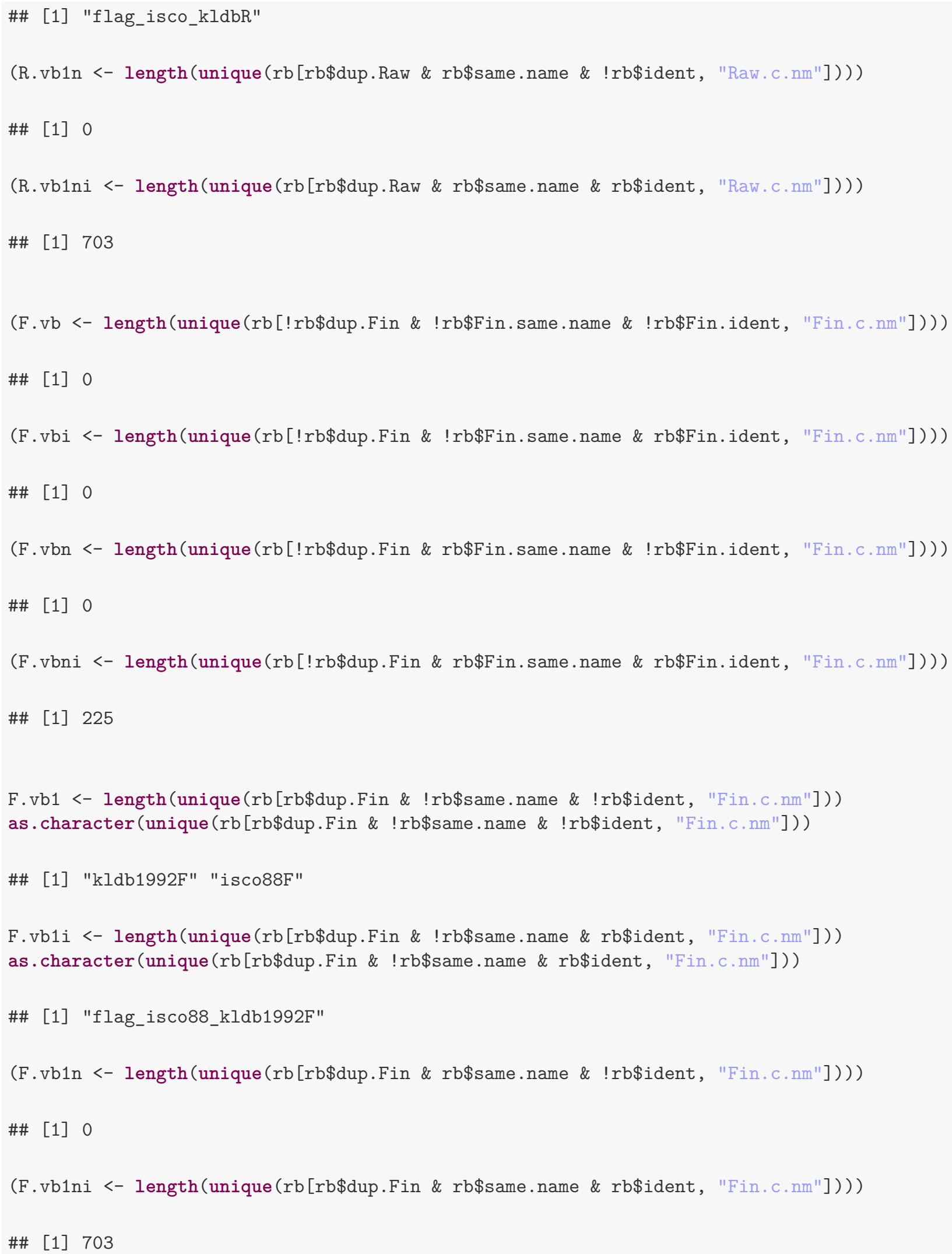




\section{A.4. comparison summary for anchor1_DD.dta Release 2.0 and Release 3.0}

\begin{tabular}{rrr} 
class & Release 2 & Release 3 \\
\hline $\mathrm{x}$ & 1 & 0 \\
$\mathrm{n}$ & 0 & 0 \\
$\mathrm{ni}$ & 423 & 423 \\
\hline $\mathrm{v}$ & 0 & 5 \\
$\mathrm{vn}$ & 43 & 43 \\
\hline $\mathrm{vb}$ & 0 & 0 \\
$\mathrm{vbi}$ & 0 & 0 \\
$\mathrm{vbn}$ & 0 & 0 \\
vbni & 225 & 225 \\
$\mathrm{vb1}$ & 2 & 2 \\
$\mathrm{vb1i}$ & 1 & 1 \\
vb1n & 0 & 0 \\
vb1ni & 703 & 703 \\
\hline sum & 1398 & 1402
\end{tabular}

Most variables are unchanged (ni, vbni, vb1ni). A small number (x, v) had been dropped or added. A number of variables (vb1, vb1i) had been (probably) renamed or recoded. A group of variables (vn) had been changed. This analysis provide no further hints for this group.

\section{A.5. selected in-depth comparison}

For illustrative purposes we present some detailed results.

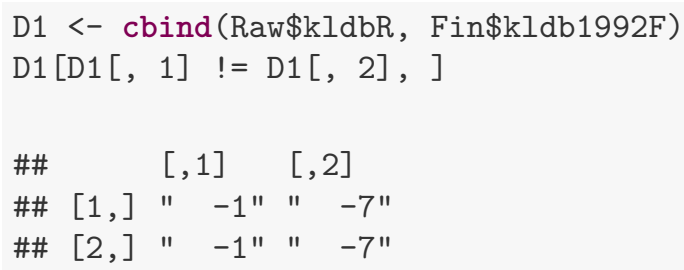

Both variables share the same number of levels. The level '-1' has been recoded to '-7'. Changing '-1' to '-7' for two individuals takes 2 Levenshtein steps (see details in [TR-2012-003]).

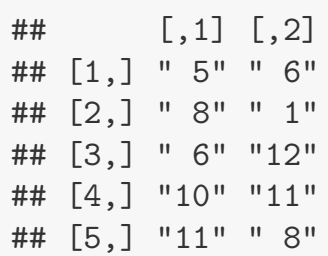

Both variables share the same number of levels. $12+12+10=34,34 / 2=17$ mappings between the levels are in use. For a bijective mapping we need 5 mappings less. Coincidentally 5 individual show a changed level. It takes 7 Levenshtein steps to make the levels equal.

D3 <- cbind (Raw\$pageR, Fin\$pageF)

$\mathrm{D} 3[\mathrm{D} 3[, 1] \quad !=\mathrm{D} 3[, 2]$, 


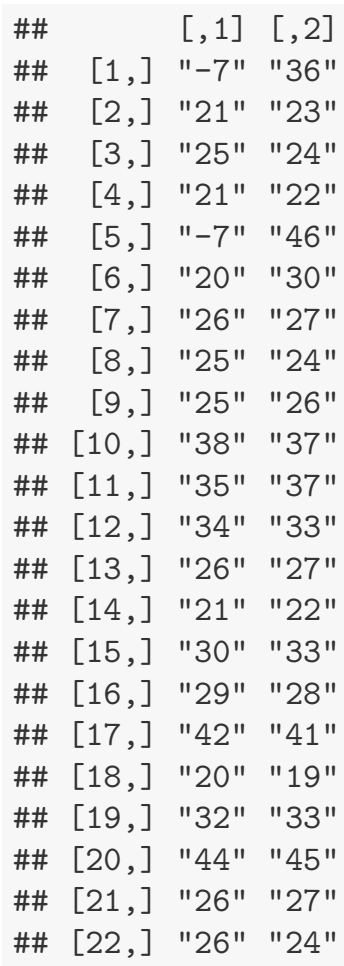

22 idividals have a new level for 'page'. It takes 25 Levenshtein steps to get them equal. There is no bijective mapping available.

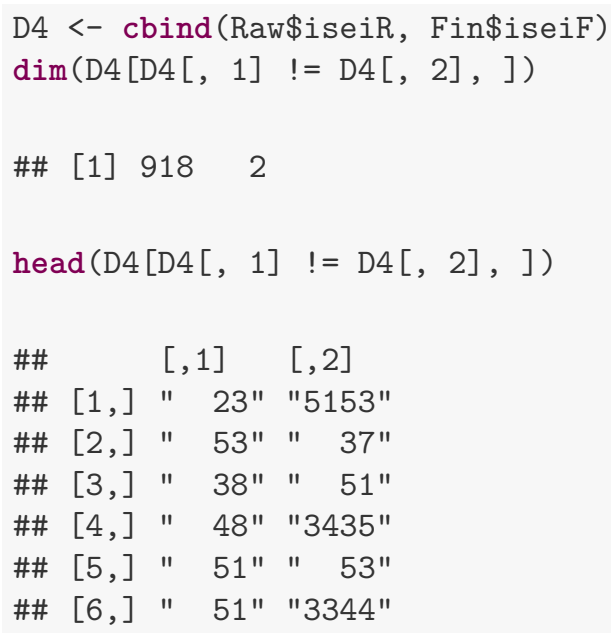

918 indiduals do have a different level. There is no bijective mapping.

\section{B. anchor2}

Using the same tools we compare the data set anchor2_dd.dta from DemoDiff Release 2.0 with the same data set from DemoDiff Release 3.0.

At first we have to load the produced data collection. Release 2.0 files are denoted with $\mathbf{R}$ and $\mathbf{R a w}$, release 3.0 files with $\mathbf{F}$ and Fin, respectively. 


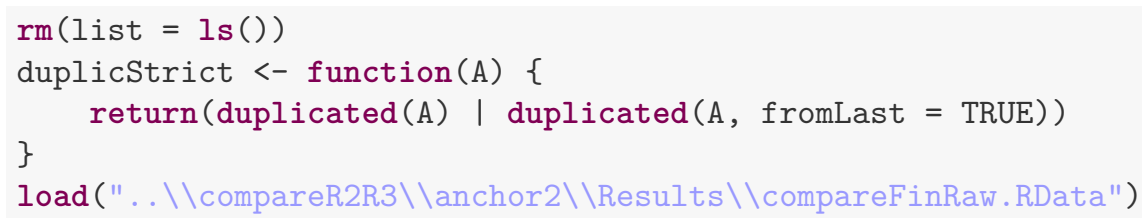

\section{B.1. no variation}

We compare the variables without variation first.

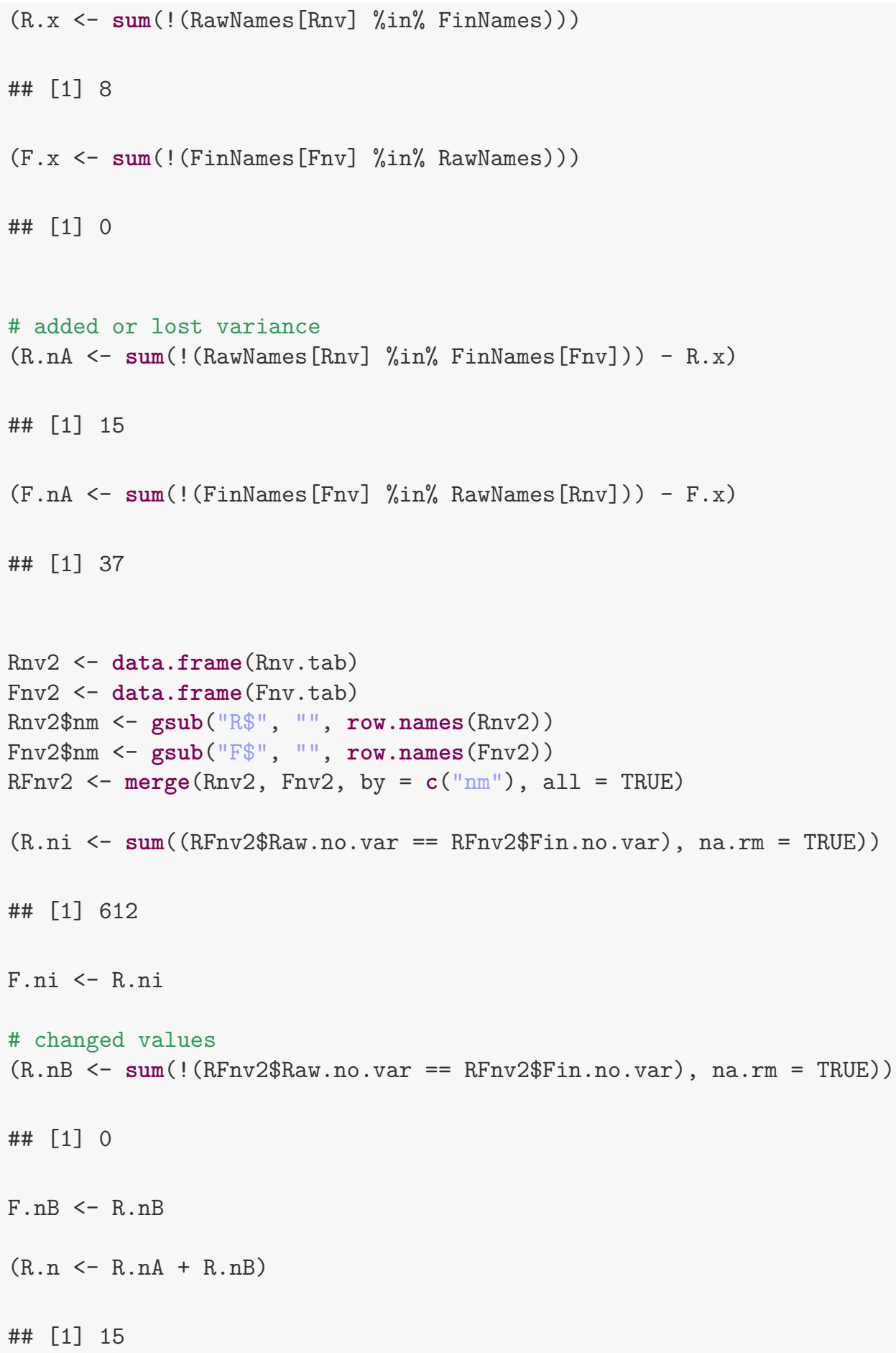


$(\mathrm{F} . \mathrm{n}<-\mathrm{F} \cdot \mathrm{nA}+\mathrm{F} \cdot \mathrm{nB})$

\#\# [1] 37

RawNames [Rnv] [! (RawNames [Rnv] \%in\% FinNames) $]$

\#\# [1] "d402" "d403" "d404" "d405" "d406" "d411" "d412" "d420"

RFnv2 [is.na(RFnv2\$Fin.no.var), 1]

\begin{tabular}{|c|c|c|c|c|c|}
\hline [1] & "d402" & "d403" & "d404" & "d405" & "d406" \\
\hline [6] & "d411" & "d412" & "d420" & "ehc27p4i1" & "ehc27p4i2" \\
\hline [11] & "ehc28p2m1" & "ehc28p2m2" & "ehc28p3m1" & "ehc28p3m2" & "ehc28p3m3" \\
\hline$[16$ & "ehc4p2m10" & "ehc4p2m11" & "ehc4p2m12" & "ehc4p2m13" & "ehc4p2m14" \\
\hline$[21$ & "ehc4p2m15" & "ehc4p2m16" & "ehc4p2m17" & & \\
\hline
\end{tabular}

FinNames [Fnv] [! (FinNames [Fnv] \%in\% RawNames) $]$

\#\# character $(0)$

RFnv2 [is.na(RFnv2\$Raw.no.var), 1]

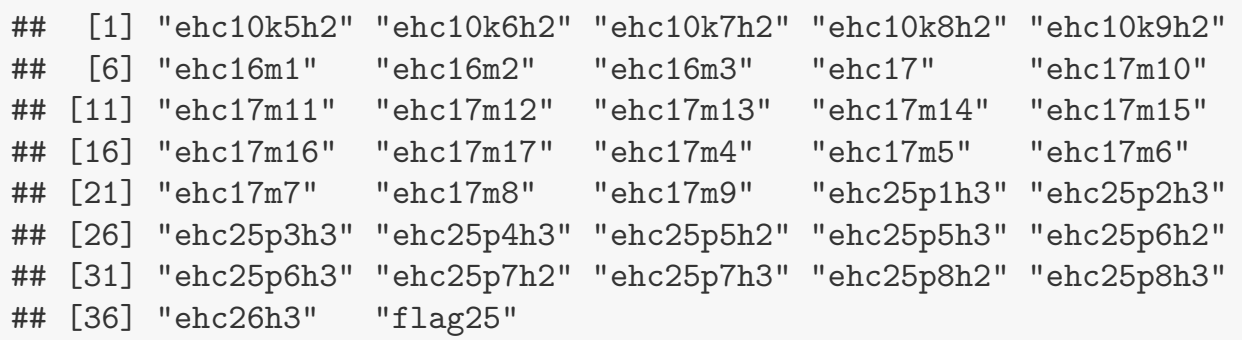

Release 2.0 For 8 variables without variation (d402, d403, d404, d405, d406, d411, d412, d420) exist no variables with the same name in the comparison data set. 612 variables without variation are identical in both data sets. 15 variables share the same name, but are not identical (got variance, changed value).

Release 3.0 612 variables without variation are identical in both data sets. 37 variables share the same name, but are not identical (lost variance, changed value).

\section{B.2. variation, but no bijective mapping}

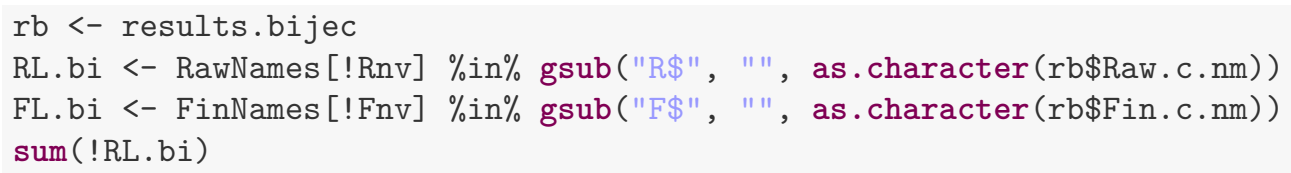


$\operatorname{sum}(! \mathrm{FL} . \mathrm{bi})$

\#\# [1] 366

$($ R.v $<-\operatorname{sum}(!($ RawNames [!Rnv] [!RL.bi] \%in\% FinNames))

\#\# [1] 1

RawNames [!Rnv] [!RL.bi] [! (RawNames [!Rnv] [!RL.bi] \%in\% FinNames)]

\#\# [1] "isco"

(R.vn $<-\operatorname{sum}($ RawNames [!Rnv] [!RL.bi] \%in\% FinNames)

\#\# [1] 369

RawNames [!Rnv] [!RL.bi] [RawNames [!Rnv] [!RL.bi] \%in\% FinNames]

\begin{tabular}{|c|c|c|c|c|c|}
\hline \#\# & [1] & "d4" & "sex" & "ehc1p1n" & "ehc1p1g" \\
\hline \#\# & [5] & "ehc2p1" & "ehc3p1" & "ehc4p1" & "ehc1p2n" \\
\hline \#\# & [9] & "ehc1p2g" & "ehc2p2" & "ehc3p2" & "ehc4p2" \\
\hline \#\# & [13] & "ehc6p2" & "ehc4p1m8" & "ehc4p1m9" & "ehc4p1m10" \\
\hline \#\# & [17] & "ehc4p1m11" & "ehc4p1m12" & "ehc4p1m13" & "ehc4p1m14" \\
\hline \#\# & [21] & "ehc4p1m15" & "ehc4p1m16" & "ehc4p1m17" & "ehc2p2m8" \\
\hline \#\# & [25] & "ehc2p2m9" & "ehc2p2m10" & "ehc2p2m11" & "ehc2p2m12" \\
\hline \#\# & [29] & "ehc2p2m13" & "ehc2p2m14" & "ehc2p2m15" & "ehc2p2m16" \\
\hline \#\# & [33] & "ehc2p2m17" & "ehc3p2m8" & "ehc3p2m9" & "ehc3p2m10" \\
\hline \#\# & [37] & "ehc3p2m11" & "ehc3p2m12" & "ehc3p2m13" & "ehc3p2m14" \\
\hline \#\# & [41] & "ehc3p2m15" & "ehc3p2m16" & "ehc3p2m17" & "ehc27p1i2" \\
\hline \#\# & [45] & "ehc28p1" & "ehc29p1" & "ehc27p2i1" & "ehc27p2i2" \\
\hline \#\# & [49] & "ehc28p2" & "ehc29p2" & "ehc27p3i1" & "ehc27p3i2" \\
\hline \#\# & [53] & "ehc28p3" & "ehc29p3" & "ehc28p1m8" & "ehc28p1m9" \\
\hline \#\# & [57] & "ehc28p1m10" & "ehc28p1m11" & "ehc28p1m12" & "ehc28p1m13" \\
\hline \#\# & [61] & "ehc28p1m14" & "ehc28p1m15" & "ehc28p1m16" & "ehc28p1m17" \\
\hline \#\# & [65] & "ehc28p2m3" & "ehc28p2m4" & "ehc28p2m5" & "ehc28p2m6" \\
\hline \#\# & [69] & "ehc28p2m7" & "ehc28p2m8" & "ehc28p2m9" & "ehc28p2m10" \\
\hline \#\# & [73] & "ehc28p2m11" & "ehc28p2m12" & "ehc28p2m13" & "ehc28p2m14" \\
\hline \#\# & {$[77]$} & "ehc28p2m15" & "ehc28p2m16" & "ehc28p2m17" & "ehc28p3m4" \\
\hline \#\# & [81] & "ehc28p3m5" & "ehc28p3m6" & "ehc28p3m7" & "ehc28p3m8" \\
\hline \#\# & [85] & "ehc28p3m9" & "ehc28p3m10" & "ehc28p3m11" & "ehc28p3m12" \\
\hline \#\# & [89] & "ehc28p3m13" & "ehc28p3m14" & "ehc28p3m15" & "ehc28p3m16" \\
\hline \#\# & [93] & "ehc28p3m17" & "ehc16m4" & "ehc16m5" & "ehc16m6" \\
\hline \#\# & [97] & "ehc16m7" & "ehc16m8" & "ehc16m9" & "ehc16m10" \\
\hline \#\# & [101] & "ehc16m11" & "ehc16m12" & "ehc16m13" & "ehc16m14" \\
\hline \#\# & [105] & "ehc16m15" & "ehc16m16" & "ehc16m17" & "ehc17m5" \\
\hline \#\# & [109] & "ehc17m6" & "ehc17m7" & "ehc17m8" & "ehc17m9" \\
\hline \#\# & [113] & "ehc17m10" & "ehc17m11" & "ehc17m12" & "ehc17m13" \\
\hline \#\# & [117] & "ehc17m14" & "ehc17m15" & "ehc17m16" & "ehc17m17" \\
\hline \#\# & [121] & "ehc16" & "ehc17" & "ehc22p1n" & "ehc22p2n" \\
\hline \#\# & [125] & "ehc22p3n" & "ehc22p4n" & "ehc22p5n" & "ehc23p1" \\
\hline \#\# & [129] & "ehc23p2" & "ehc23p3" & "ehc23p4" & "ehc23p5" \\
\hline \#\# & [133] & "ehc24p1m" & "ehc24p2m" & "ehc24p3m" & "ehc24p4m" \\
\hline \#\# & [137] & "ehc24p5m" & "ehc24p6m" & "ehc24p1y" & "ehc24p2y" \\
\hline
\end{tabular}




\begin{tabular}{|c|c|c|c|c|c|}
\hline \#\# & [141] & "ehc24p3y" & "ehc24p4y" & "ehc24p5y" & "ehc24p6y" \\
\hline \#\# & [145] & "ehc25p1h1" & "ehc25p2h1" & "ehc25p3h1" & "ehc25p4h1" \\
\hline \#\# & [149] & "ehc25p5h1" & "ehc25p1h2" & "ehc25p2h2" & "ehc25p3h2" \\
\hline \#\# & [153] & "ehc25p4h2" & "ehc25p5h2" & "ehc25p7h2" & "ehc25p1h3" \\
\hline \#\# & [157] & "ehc25p2h3" & "ehc25p3h3" & "ehc25p4h3" & "ehc25p5h3" \\
\hline \#\# & [161] & "ehc25p7h3" & "ehc26h1" & "ehc26h2" & "ehc26h3" \\
\hline \#\# & [165] & "ehc10k1h1" & "ehc10k2h1" & "ehc10k3h1" & "ehc10k4h1" \\
\hline \#\# & [169] & "ehc10k5h1" & "ehc10k6h1" & "ehc10k7h1" & "ehc10k1h2" \\
\hline \#\# & [173] & "ehc10k2h2" & "ehc10k3h2" & "ehc10k4h2" & "hhnr" \\
\hline \#\# & [177] & "hh1nr" & "hh2nr" & "hh1a" & "hh2a" \\
\hline \#\# & [181] & "hh11" & "hh21" & "hh1t0" & "hh2t0" \\
\hline \#\# & [185] & "hp" & "hpnr" & "hpn" & "hpg" \\
\hline \#\# & [189] & "hpo" & "hpsep" & "hpmt" & "hpm" \\
\hline \#\# & [193] & "hpc" & "hps2" & "hp0nr" & "hp0n" \\
\hline \#\# & [197] & "hp0g" & "hp0cto" & "hc6h1" & "hc6h2" \\
\hline \#\# & [201] & "job17" & "job18h" & "sat2" & "sin3i1" \\
\hline \#\# & [205] & "sin3i2" & "sin1" & "sin2" & "sin6i1" \\
\hline \#\# & [209] & "sin6i2" & "sin6i3" & "sin6i4" & "sin6i5" \\
\hline \#\# & [213] & "pa2m" & "pa30" & "pa3" & "sdp1d" \\
\hline \#\# & [217] & "sdp1m" & "sdp1y" & "sdp2i1" & "sdp2i2" \\
\hline \#\# & [221] & "sdp2i3" & "sdp2i4" & "sdp2i5" & "sdp2i6" \\
\hline \#\# & [225] & "sdp2i7" & "sdp2i8" & "sdp2i9" & "sdp2i10" \\
\hline \#\# & [229] & "sdp2i11" & "sdp2i12" & "sdp3" & "sdp6" \\
\hline \#\# & [233] & "sdp21" & "sdp22" & "sdp10i16" & "sdp10i17" \\
\hline \#\# & [237] & "sdp10i7" & "sdp10i18" & "sdp10i19" & "sdp10i9" \\
\hline \#\# & [241] & "sdp10i20" & "sdp10i21" & "sdp10i22" & "sdp10i1" \\
\hline \#\# & [245] & "sdp10i2" & "sdp10i3" & "sdp10i4" & "sdp10i5" \\
\hline \#\# & [249] & "sdp10i6" & "sdp10i13" & "sdp10i8" & "sdp10i10" \\
\hline \#\# & [253] & "sdp10i11" & "sdp10i12" & "sdp10i15" & "sdp10i14" \\
\hline \#\# & [257] & "sdp16" & "sdp17" & "sdp18" & "hcp1i1" \\
\hline \#\# & [261] & "hcp1i2" & "hcp2" & "hcp3h" & "hcp3m" \\
\hline \#\# & [265] & "hcp4h" & "hcp5" & "pa9" & "pa11" \\
\hline \#\# & [269] & "pa13" & "pa14i1" & "pa14i2" & "pa14i3" \\
\hline \#\# & [273] & "pa14i4" & "pa14i5" & "pa15" & "cps1" \\
\hline \#\# & [277] & "sat3" & "pa26" & "pa27" & "pa28" \\
\hline \#\# & [281] & "sep1i1" & "sep1i2" & "sep2" & "sep18" \\
\hline \#\# & [285] & "sep12" & "sep13" & "sep14" & "sep17i1" \\
\hline \#\# & [289] & "sep17i2" & "sep17i3" & "sep17i4" & "sep16" \\
\hline \#\# & [293] & "frt2" & "frt11v1i6" & "frt11v1i7" & "frt11v1i8" \\
\hline \#\# & [297] & "frt11v2i6" & "frt11v2i7" & "frt11v2i8" & "crn22p1" \\
\hline \#\# & [301] & "crn33p1i1" & "crn33p1i2" & "crn33p1i3" & "crn34p1" \\
\hline \#\# & [305] & "igr22" & "igr25" & "igr28" & "igr39p3" \\
\hline \#\# & [309] & "igr40p3" & "igr41p1" & "rtr43" & "inc24" \\
\hline \#\# & [313] & "inc26i2" & "inc26i3" & "inc27i2" & "inc27i3" \\
\hline \#\# & [317] & "int4i6" & "lmhh1" & "lmhh2" & "lvhh1" \\
\hline \#\# & [321] & "Ivhh2" & "avhh1" & "hv1" & "tag_sex" \\
\hline \#\# & [325] & "tag_dobp" & "flag25" & "sex_gen" & "psex_gen" \\
\hline \#\# & [329] & "pdoby_gen" & "pdobm_gen" & "page" & "mage" \\
\hline \#\# & [333] & "k1age" & "k2age" & "pcob" & "pnat1" \\
\hline \#\# & [337] & "marstat" & "np" & "ncoh" & "nmar" \\
\hline \#\# & [341] & "meetdur" & "reldur" & "cohabdur" & "mardur" \\
\hline \#\# & [345] & "homosex" & "infertile" & "pregnant" & "pnkidsbioalv" \\
\hline \#\# & [349] & "res2nd" & "hhsizemrd" & "mmrd" & "fmrd" \\
\hline \#\# & [353] & "childmrd" & "othmrd" & "hhcomp" & "penrol" \\
\hline \#\# & [357] & "pschool" & "pvocat" & "pisced" & "pisced2" \\
\hline \#\# & [361] & "pcasmin" & "pyeduc" & "egp" & "isei" \\
\hline
\end{tabular}



\#\# [365] "siops"
"hhincgcee"
"pcasprim"
"pcassec"
\#\# [369] "plfs"

$($ F.v $<-\operatorname{sum}(!($ FinNames $[$ !Fnv] [!FL.bi] \%in\% RawNames $))$ )

\#\# [1] 7

FinNames [!Fnv] [!FL.bi] [! (FinNames [!Fnv] [!FL.bi] \%in\% RawNames) ]

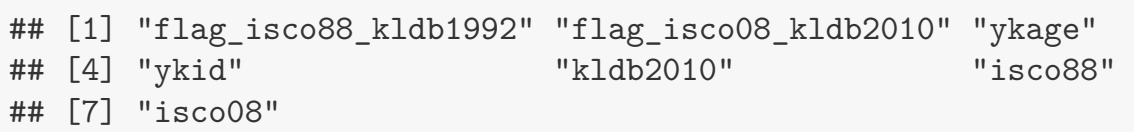

(F.vn $<-\operatorname{sum}($ FinNames [!Fnv] [!FL.bi] \%in\% RawNames))

\#\# [1] 359

FinNames [!Fnv] [!FL.bi] [FinNames [!Fnv] [!FL.bi] \%in\% RawNames]

\begin{tabular}{|c|c|c|c|c|c|}
\hline \#\# & [1] & "d4" & "sex" & "ehc1p1n" & "ehc1p1g" \\
\hline \#\# & [5] & "ehc2p1" & "ehc3p1" & "ehc4p1" & "ehc1p2n" \\
\hline \#\# & [9] & "ehc1p2g" & "ehc2p2" & "ehc3p2" & "ehc4p2" \\
\hline \#\# & [13] & "ehc6p2" & "ehc4p1m8" & "ehc4p1m9" & "ehc4p1m10" \\
\hline \#\# & [17] & "ehc4p1m11" & "ehc4p1m12" & "ehc4p1m13" & "ehc4p1m14" \\
\hline \#\# & [21] & "ehc4p1m15" & "ehc4p1m16" & "ehc4p1m17" & "ehc2p2m8" \\
\hline \#\# & [25] & "ehc2p2m9" & "ehc2p2m10" & "ehc2p2m11" & "ehc2p2m12" \\
\hline \#\# & [29] & "ehc2p2m13" & "ehc2p2m14" & "ehc2p2m15" & "ehc2p2m16" \\
\hline \#\# & [33] & "ehc2p2m17" & "ehc3p2m8" & "ehc3p2m9" & "ehc3p2m10" \\
\hline \#\# & [37] & "ehc3p2m11" & "ehc3p2m12" & "ehc3p2m13" & "ehc3p2m14" \\
\hline \#\# & [41] & "ehc3p2m15" & "ehc3p2m16" & "ehc3p2m17" & "ehc4p2m8" \\
\hline \#\# & [45] & "ehc4p2m9" & "ehc4p2m10" & "ehc4p2m11" & "ehc4p2m12" \\
\hline \#\# & [49] & "ehc4p2m13" & "ehc4p2m14" & "ehc4p2m15" & "ehc4p2m16" \\
\hline \#\# & [53] & "ehc4p2m17" & "ehc27p1i2" & "ehc28p1" & "ehc29p1" \\
\hline \#\# & [57] & "ehc27p2i1" & "ehc27p2i2" & "ehc28p2" & "ehc29p2" \\
\hline \#\# & [61] & "ehc27p3i1" & "ehc27p3i2" & "ehc28p3" & "ehc29p3" \\
\hline \#\# & [65] & "ehc27p4i1" & "ehc27p4i2" & "ehc28p1m8" & "ehc28p1m9" \\
\hline \#\# & [69] & "ehc28p1m10" & "ehc28p1m11" & "ehc28p1m12" & "ehc28p1m13" \\
\hline \#\# & [73] & "ehc28p1m14" & "ehc28p1m15" & "ehc28p1m16" & "ehc28p1m17" \\
\hline \#\# & {$[77]$} & "ehc28p2m1" & "ehc28p2m2" & "ehc28p2m3" & "ehc28p2m4" \\
\hline \#\# & [81] & "ehc28p2m5" & "ehc28p2m6" & "ehc28p2m7" & "ehc28p2m8" \\
\hline \#\# & [85] & "ehc28p2m9" & "ehc28p2m10" & "ehc28p2m11" & "ehc28p2m12" \\
\hline \#\# & [89] & "ehc28p2m13" & "ehc28p2m14" & "ehc28p2m15" & "ehc28p2m16" \\
\hline \#\# & [93] & "ehc28p2m17" & "ehc28p3m1" & "ehc28p3m2" & "ehc28p3m3" \\
\hline \#\# & [97] & "ehc28p3m4" & "ehc28p3m5" & "ehc28p3m6" & "ehc28p3m7" \\
\hline \#\# & [101] & "ehc28p3m8" & "ehc28p3m9" & "ehc28p3m10" & "ehc28p3m11" \\
\hline \#\# & [105] & "ehc28p3m12" & "ehc28p3m13" & "ehc28p3m14" & "ehc28p3m15" \\
\hline \#\# & [109] & "ehc28p3m16" & "ehc28p3m17" & "ehc16m5" & "ehc16m6" \\
\hline \#\# & [113] & "ehc16m7" & "ehc16m8" & "ehc16m9" & "ehc16m10" \\
\hline \#\# & [117] & "ehc16m11" & "ehc16m12" & "ehc16m13" & "ehc16m14" \\
\hline \#\# & [121] & "ehc16m15" & "ehc16m16" & "ehc16m17" & "ehc16" \\
\hline \#\# & [125] & "ehc22p1n" & "ehc22p2n" & "ehc22p3n" & "ehc22p4n" \\
\hline \#\# & [129] & "ehc22p5n" & "ehc23p1" & "ehc23p2" & "ehc23p3" \\
\hline \#\# & [133] & "ehc23p4" & "ehc23p5" & "ehc24p1m" & "ehc24p2m" \\
\hline
\end{tabular}




\begin{tabular}{|c|c|c|c|c|c|}
\hline \#\# & {$[137]$} & "ehc24p3m" & "ehc24p4m" & "ehc24p5m" & "ehc24p7m" \\
\hline \#\# & [141] & "ehc24p1y" & "ehc24p2y" & "ehc24p3y" & "ehc24p4y" \\
\hline \#\# & [145] & "ehc24p5y" & "ehc24p7y" & "ehc25p1h1" & "ehc25p2h1" \\
\hline \#\# & [149] & "ehc25p3h1" & "ehc25p4h1" & "ehc25p5h1" & "ehc25p7h1" \\
\hline \#\# & [153] & "ehc25p1h2" & "ehc25p2h2" & "ehc25p3h2" & "ehc26h1" \\
\hline \#\# & {$[157]$} & "ehc26h2" & "ehc10k1h1" & "ehc10k2h1" & "ehc10k3h1" \\
\hline \#\# & [161] & "ehc10k4h1" & "ehc10k5h1" & "ehc10k6h1" & "ehc10k7h1" \\
\hline \#\# & [165] & "ehc10k1h2" & "ehc10k2h2" & "ehc10k3h2" & "ehc10k4h2" \\
\hline \#\# & [169] & "hhnr" & "hh1nr" & "hh2nr" & "hh1a" \\
\hline \#\# & [173] & "hh2a" & "hh11" & "hh21" & "hh1t0" \\
\hline \#\# & {$[177]$} & "hh2t0" & "hp" & "hpnr" & "hpn" \\
\hline \#\# & [181] & "hpg" & "hpo" & "hpsep" & "hpmt" \\
\hline \#\# & [185] & "hpm" & "hpc" & "hps2" & "hp0nr" \\
\hline \#\# & [189] & "hp0n" & "hp0g" & "hpocto" & "hc6h1" \\
\hline \#\# & [193] & "hc6h2" & "job17" & "job18h" & "sat2" \\
\hline \#\# & [197] & "sin3i1" & "sin3i2" & "sin1" & "sin2" \\
\hline \#\# & [201] & "sin6i1" & "sin6i2" & "sin6i3" & "sin6i4" \\
\hline \#\# & [205] & "sin6i5" & "pa2m" & "pa30" & "pa3" \\
\hline \#\# & [209] & "sdp1d" & "sdp1m" & "sdp1y" & "sdp2i1" \\
\hline \#\# & [213] & "sdp2i2" & "sdp2i3" & "sdp2i4" & "sdp2i5" \\
\hline \#\# & [217] & "sdp2i6" & "sdp2i7" & "sdp2i8" & "sdp2i9" \\
\hline \#\# & [221] & "sdp2i10" & "sdp2i11" & "sdp2i12" & "sdp3" \\
\hline \#\# & [225] & "sdp6" & "sdp21" & "sdp22" & "sdp10i16" \\
\hline \#\# & [229] & "sdp10i17" & "sdp10i7" & "sdp10i18" & "sdp10i19" \\
\hline \#\# & [233] & "sdp10i9" & "sdp10i20" & "sdp10i21" & "sdp10i22" \\
\hline \#\# & {$[237]$} & "sdp10i1" & "sdp10i2" & "sdp10i3" & "sdp10i4" \\
\hline \#\# & [241] & "sdp10i5" & "sdp10i6" & "sdp10i13" & "sdp10i8" \\
\hline \#\# & [245] & "sdp10i10" & "sdp10i11" & "sdp10i12" & "sdp10i15" \\
\hline \#\# & [249] & "sdp10i14" & "sdp16" & "sdp17" & "sdp18" \\
\hline \#\# & [253] & "hcp1i1" & "hcp1i2" & "hсp2" & "hсp3h" \\
\hline \#\# & [257] & "hcp3m" & "hcp5" & "pa9" & "pa11" \\
\hline \#\# & [261] & "pa13" & "pa14i1" & "pa14i2" & "pa14i3" \\
\hline \#\# & [265] & "pa14i4" & "pa14i5" & "pa15" & "cps1" \\
\hline \#\# & [269] & "sat3" & "pa26" & "pa27" & "pa28" \\
\hline \#\# & [273] & "sep1i1" & "sep1i2" & "sep2" & "sep18" \\
\hline \#\# & [277] & "sep12" & "sep13" & "sep14" & "sep17i1" \\
\hline \#\# & [281] & "sep17i2" & "sep17i3" & "sep17i4" & "sep16" \\
\hline \#\# & [285] & "frt2" & "frt11v1i6" & "frt11v1i7" & "frt11v1i8" \\
\hline \#\# & [289] & "frt11v2i6" & "frt11v2i7" & "frt11v2i8" & "crn22p1" \\
\hline \#\# & [293] & "crn33p1i1" & "crn33p1i2" & "crn33p1i3" & "crn34p1" \\
\hline \#\# & [297] & "igr22" & "igr25" & "igr28" & "igr39p3" \\
\hline \#\# & [301] & "igr40p3" & "igr41p1" & "rtr43" & "inc24" \\
\hline \#\# & [305] & "inc26i2" & "inc26i3" & "inc27i2" & "inc27i3" \\
\hline \#\# & [309] & "int4i6" & "lmhh1" & "lmhh2" & "Ivhh1" \\
\hline \#\# & [313] & "lvhh2" & "hv1" & "tag_sex" & "tag_dobp" \\
\hline \#\# & [317] & "sex_gen" & "psex_gen" & "pdoby_gen" & "pdobm_gen" \\
\hline \#\# & [321] & "page" & "mage" & "k1age" & "k2age" \\
\hline \#\# & [325] & "pcob" & "pnat1" & "marstat" & "np" \\
\hline \#\# & [329] & "ncoh" & "nmar" & "meetdur" & "reldur" \\
\hline \#\# & [333] & "cohabdur" & "mardur" & "homosex" & "infertile" \\
\hline \#\# & [337] & "pregnant" & "pnkidsbioalv" & "res2nd" & "hhsizemrd" \\
\hline \#\# & [341] & "mmrd" & "fmrd" & "childmrd" & "othmrd" \\
\hline \#\# & [345] & "hhcomp" & "penrol" & "pschool" & "pvocat" \\
\hline \#\# & [349] & "pisced" & "pisced2" & "pcasmin" & "pyeduc" \\
\hline \#\# & [353] & "egp" & "isei" & "siops" & "hhincgcee" \\
\hline \#\# & [357] & "pcasprim" & "pcassec" & "plfs" & \\
\hline
\end{tabular}


Release 2.0 For 1 variables with variation but without any bijective mapping exist no variables with the same name in the Release 3.0 data. 369 variables share the name with one of the variables in Release 3.0 at least.

Release 3.0 For 7 variables with variation but without any bijective mapping exist no variables with the same name in the Release 2.0 data. 359 variables share the name with one of the variables in Release 2.0 at least.

We compare all variables pairs (with variation) which share the same name but are not connected with a bijective mapping. Those variables imply different information. DemoDiff data user should check whether they are using those variables for possible effects on their research. The number of pairs may differ from 369 and 359 .

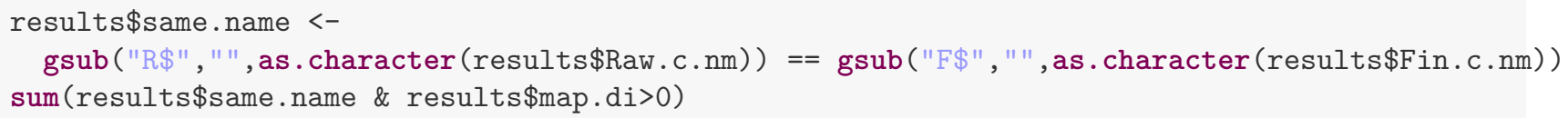

\begin{tabular}{|c|c|c|c|c|c|c|}
\hline \#\# & Fin.c.nm & Fin.c.ls & Raw.c.nm & Raw.c.ls & $\operatorname{map} \cdot d i$ & ed.di.sum \\
\hline \#\# & $\mathrm{d} 4 \mathrm{~F}$ & 2 & $d 4 R$ & 2 & 2 & 1 \\
\hline \#\# & $\operatorname{sexF}$ & 2 & sexR & 2 & 2 & 1 \\
\hline \#\# & ehc1p1nF & 3 & ehc1p1nR & 3 & 2 & 16 \\
\hline \#\# & ehc1p1gF & 3 & ehc1p1gR & 3 & 4 & 14 \\
\hline \#\# & ehc2p1F & 3 & ehc2p1R & 3 & 2 & 24 \\
\hline \#\# & ehc3p1F & 3 & ehc3p1R & 3 & 2 & 22 \\
\hline \#\# & ehc4p1F & 3 & ehc4p1R & 3 & 4 & 21 \\
\hline \#\# & ehc1p2nF & 2 & ehc1p $2 \mathrm{nR}$ & 2 & 2 & 2 \\
\hline \#\# & ehc1p2gF & 3 & ehc1p2gR & 3 & 2 & 2 \\
\hline \#\# & ehc2p2F & 3 & ehc2p2R & 3 & 2 & 2 \\
\hline \#\# & ehc3p2F & 3 & ehc $3 p 2 R$ & 3 & 2 & 2 \\
\hline \#\# & ehc4p2F & 2 & ehc4p2R & 2 & 2 & 2 \\
\hline \#\# & ehc6p2F & 2 & ehc6p2R & 2 & 2 & 2 \\
\hline \#\# & ehc4p1m8F & 3 & ehc4p1m8R & 3 & 2 & 1 \\
\hline \#\# & ehc4p1m9F & 3 & ehc4p1m9R & 3 & 2 & 1 \\
\hline \#\# & ehc4p1m10F & 2 & ehc4p1m10R & 2 & 2 & 1 \\
\hline \#\# & ehc4p1m11F & 2 & ehc4p1m11R & 2 & 2 & 1 \\
\hline \#\# & ehc4p1m12F & 2 & ehc4p1m12R & 2 & 2 & 1 \\
\hline \#\# & ehc4p1m13F & 2 & ehc4p1m13R & 2 & 2 & 1 \\
\hline \#\# & ehc4p1m14F & 2 & ehc4p1m14R & 2 & 2 & 1 \\
\hline \#\# & ehc4p1m15F & 2 & ehc4p1m15R & 2 & 2 & 1 \\
\hline \#\# & ehc4p1m16F & 2 & ehc4p1m16R & 2 & 2 & 1 \\
\hline \#\# & ehc4p1m17F & 2 & ehc4p1m17R & 2 & 2 & 1 \\
\hline \#\# & ehc $2 p 2 m 8 F$ & 3 & ehc $2 p 2 m 8 R$ & 3 & 2 & 2 \\
\hline \#\# & ehc2p $2 \mathrm{~m} 9 \mathrm{~F}$ & 3 & ehc $2 \mathrm{p} 2 \mathrm{~m} 9 \mathrm{R}$ & 3 & 2 & 2 \\
\hline \#\# & ehc $2 \mathrm{p} 2 \mathrm{~m} 10 \mathrm{~F}$ & 3 & ehc $2 \mathrm{p} 2 \mathrm{~m} 10 \mathrm{R}$ & 2 & 1 & 1174 \\
\hline \#\# & ehc $2 p 2 m 11 F$ & 3 & ehc $2 p 2 m 11 R$ & 2 & 1 & 1174 \\
\hline \#\# & ehc $2 \mathrm{p} 2 \mathrm{~m} 12 \mathrm{~F}$ & 3 & ehc $2 p 2 m 12 R$ & 2 & 1 & 1174 \\
\hline \#\# & ehc $2 \mathrm{p} 2 \mathrm{~m} 13 \mathrm{~F}$ & 3 & ehc $2 p 2 m 13 R$ & 2 & 1 & 1174 \\
\hline \#\# & ehc $2 \mathrm{p} 2 \mathrm{~m} 14 \mathrm{~F}$ & 3 & ehc $2 p 2 m 14 R$ & 2 & 1 & 1174 \\
\hline \#\# & ehc $2 p 2 m 15 F$ & 3 & ehc $2 p 2 m 15 R$ & 2 & 1 & 1174 \\
\hline \#\# & ehc $2 \mathrm{p} 2 \mathrm{~m} 16 \mathrm{~F}$ & 3 & ehc $2 p 2 m 16 R$ & 2 & 1 & 1174 \\
\hline \#\# & ehc $2 p 2 m 17 F$ & 3 & ehc $2 p 2 m 17 R$ & 2 & 1 & 1174 \\
\hline
\end{tabular}




\begin{tabular}{|c|c|c|c|c|c|c|}
\hline \#\# & ehc3p $2 \mathrm{~m} 8 \mathrm{~F}$ & 2 & ehc3p2m8R & 3 & 1 & 2 \\
\hline \#\# & ehc3p $2 \mathrm{~m} 9 \mathrm{~F}$ & 3 & ehc3p2m9R & 3 & 2 & 2 \\
\hline \#\# & ehc3p $2 \mathrm{~m} 10 \mathrm{~F}$ & 3 & ehc $3 p 2 m 10 R$ & 2 & 1 & 1174 \\
\hline \#\# & ehc3p $2 \mathrm{~m} 11 \mathrm{~F}$ & 3 & ehc $3 p 2 m 11 R$ & 2 & 1 & 1174 \\
\hline \#\# & ehc3p $2 \mathrm{~m} 12 \mathrm{~F}$ & 3 & ehc $3 p 2 m 12 R$ & 2 & 1 & 1174 \\
\hline \#\# & ehc3p $2 m 13 F$ & 3 & ehc3p $2 m 13 R$ & 2 & 1 & 1174 \\
\hline \#\# & ehc3p $2 \mathrm{~m} 14 \mathrm{~F}$ & 3 & ehc3p $2 \mathrm{~m} 14 \mathrm{R}$ & 2 & 1 & 1174 \\
\hline \#\# & ehc3p $2 m 15 F$ & 3 & ehc3p 2 m15R & 2 & 1 & 1174 \\
\hline \#\# & ehc3p $2 \mathrm{~m} 16 \mathrm{~F}$ & 3 & ehc3p2m16R & 2 & 1 & 1174 \\
\hline \#\# & ehc3p $2 m 17 F$ & 3 & ehc $3 p 2 m 17 R$ & 2 & 1 & 1174 \\
\hline \#\# & ehc4p2m8F & 2 & ehc4p2m8R & 2 & 2 & 2 \\
\hline \#\# & ehc4p $2 \mathrm{~m} 9 \mathrm{~F}$ & 2 & ehc4p2m9R & 2 & 2 & 2 \\
\hline \#\# & ehc27p1i2F & 9 & ehc27p1i2R & 8 & 1 & 2 \\
\hline \#\# & ehc28p1F & 2 & ehc28p1R & 2 & 2 & 12 \\
\hline \#\# & ehc29p1F & 3 & ehc29p1R & 3 & 4 & 19 \\
\hline \#\# & ehc27p2i1F & 3 & ehc27p2i1R & 2 & 3 & 33 \\
\hline \#\# & ehc $27 p 2 i 2 F$ & 16 & ehc27p2i2R & 15 & 11 & 34 \\
\hline \#\# & ehc28p2F & 4 & ehc28p2R & 3 & 5 & 33 \\
\hline \#\# & ehc29p2F & 4 & ehc29p2R & 3 & 1 & 8 \\
\hline \#\# & ehc27p3i1F & 3 & ehc27p3i1R & 2 & 1 & 4 \\
\hline \#\# & ehc27p3i2F & 6 & ehc27p3i2R & 5 & 1 & 4 \\
\hline \#\# & ehc28p3F & 4 & ehc28p3R & 3 & 1 & 4 \\
\hline \#\# & ehc29p3F & 3 & ehc29p3R & 2 & 1 & 3 \\
\hline \#\# & ehc28p1m8F & 3 & ehc28p1m8R & 3 & 2 & 1 \\
\hline \#\# & ehc28p1m9F & 3 & ehc28p1m9R & 3 & 2 & 2 \\
\hline \#\# & ehc $28 \mathrm{p} 1 \mathrm{~m} 10 \mathrm{~F}$ & 2 & ehc $28 \mathrm{p} 1 \mathrm{~m} 10 \mathrm{R}$ & 2 & 2 & 4 \\
\hline \#\# & ehc $28 \mathrm{p} 1 \mathrm{~m} 11 \mathrm{~F}$ & 2 & ehc $28 p 1 m 11 R$ & 2 & 2 & 4 \\
\hline \#\# & ehc28p1m12F & 2 & ehc $28 \mathrm{p} 1 \mathrm{~m} 12 \mathrm{R}$ & 2 & 2 & 5 \\
\hline \#\# & ehc28p1m13F & 2 & ehc $28 \mathrm{p} 1 \mathrm{~m} 13 \mathrm{R}$ & 2 & 2 & 9 \\
\hline \#\# & ehc28p1m14F & 2 & ehc28p1m14R & 2 & 2 & 10 \\
\hline \#\# & ehc $28 \mathrm{p} 1 \mathrm{~m} 15 \mathrm{~F}$ & 2 & ehc $28 p 1 m 15 R$ & 2 & 2 & 10 \\
\hline \#\# & ehc $28 \mathrm{p} 1 \mathrm{~m} 16 \mathrm{~F}$ & 2 & ehc $28 \mathrm{p} 1 \mathrm{~m} 16 \mathrm{R}$ & 2 & 2 & 11 \\
\hline \#\# & ehc $28 \mathrm{p} 1 \mathrm{~m} 17 \mathrm{~F}$ & 2 & ehc28p1m17R & 2 & 2 & 12 \\
\hline \#\# & ehc28p2m3F & 3 & ehc28p2m3R & 2 & 1 & 3 \\
\hline \#\# & ehc $28 p 2 m 4 F$ & 4 & ehc $28 p 2 m 4 R$ & 3 & 3 & 5 \\
\hline \#\# & ehc $28 \mathrm{p} 2 \mathrm{~m} 5 \mathrm{~F}$ & 4 & ehc $28 p 2 m 5 R$ & 3 & 3 & 16 \\
\hline \#\# & ehc $28 \mathrm{p} 2 \mathrm{~m} 6 \mathrm{~F}$ & 4 & ehc $28 p 2 m 6 R$ & 3 & 3 & 16 \\
\hline \#\# & ehc $28 p 2 m 7 F$ & 4 & ehc28p2m7R & 3 & 3 & 22 \\
\hline \#\# & ehc $28 \mathrm{p} 2 \mathrm{~m} 8 \mathrm{~F}$ & 4 & ehc $28 \mathrm{p} 2 \mathrm{~m} 8 \mathrm{R}$ & 3 & 5 & 28 \\
\hline \#\# & ehc28p2m9F & 4 & ehc28p2m9R & 3 & 5 & 33 \\
\hline \#\# & ehc $28 \mathrm{p} 2 \mathrm{~m} 10 \mathrm{~F}$ & 4 & ehc $28 \mathrm{p} 2 \mathrm{~m} 10 \mathrm{R}$ & 3 & 5 & 33 \\
\hline \#\# & ehc $28 \mathrm{p} 2 \mathrm{~m} 11 \mathrm{~F}$ & 4 & ehc $28 \mathrm{p} 2 \mathrm{~m} 11 \mathrm{R}$ & 3 & 5 & 33 \\
\hline \#\# & ehc $28 \mathrm{p} 2 \mathrm{~m} 12 \mathrm{~F}$ & 4 & ehc $28 \mathrm{p} 2 \mathrm{~m} 12 \mathrm{R}$ & 3 & 5 & 33 \\
\hline \#\# & ehc $28 \mathrm{p} 2 \mathrm{~m} 13 \mathrm{~F}$ & 4 & ehc $28 \mathrm{p} 2 \mathrm{~m} 13 \mathrm{R}$ & 3 & 5 & 33 \\
\hline \#\# & ehc $28 \mathrm{p} 2 \mathrm{~m} 14 \mathrm{~F}$ & 4 & ehc28p2m14R & 3 & 5 & 33 \\
\hline \#\# & ehc $28 \mathrm{p} 2 \mathrm{~m} 15 \mathrm{~F}$ & 4 & ehc $28 p 2 m 15 R$ & 3 & 5 & 33 \\
\hline \#\# & ehc $28 \mathrm{p} 2 \mathrm{~m} 16 \mathrm{~F}$ & 4 & ehc $28 p 2 m 16 R$ & 3 & 5 & 33 \\
\hline \#\# & ehc $28 \mathrm{p} 2 \mathrm{~m} 17 \mathrm{~F}$ & 4 & ehc $28 p 2 m 17 R$ & 3 & 5 & 33 \\
\hline \#\# & ehc28p3m4F & 3 & ehc28p3m4R & 2 & 1 & 2 \\
\hline \#\# & ehc $28 \mathrm{p} 3 \mathrm{~m} 5 \mathrm{~F}$ & 3 & ehc28p3m5R & 2 & 1 & 3 \\
\hline \#\# & ehc $28 p 3 m 6 F$ & 3 & ehc28p3m6R & 2 & 1 & 4 \\
\hline \#\# & ehc $28 p 3 m 7 F$ & 4 & ehc28p3m7R & 3 & 1 & 4 \\
\hline \#\# & ehc $28 \mathrm{p} 3 \mathrm{~m} 8 \mathrm{~F}$ & 4 & ehc28p3m8R & 3 & 1 & 4 \\
\hline \#\# & ehc28p3m9F & 4 & ehc28p3m9R & 3 & 1 & 4 \\
\hline \#\# & ehc $28 \mathrm{p} 3 \mathrm{~m} 10 \mathrm{~F}$ & 4 & ehc $28 \mathrm{p} 3 \mathrm{~m} 10 \mathrm{R}$ & 3 & 1 & 4 \\
\hline \#\# & ehc $28 \mathrm{p} 3 \mathrm{~m} 11 \mathrm{~F}$ & 4 & ehc $28 p 3 m 11 R$ & 3 & 1 & 4 \\
\hline
\end{tabular}




\begin{tabular}{|c|c|c|c|c|c|c|}
\hline \#\# & ehc28p3m12F & 4 & ehc28p3m12R & 3 & 1 & 4 \\
\hline \#\# & ehc $28 p 3 m 13 F$ & 4 & ehc 28 p3m13R & 3 & 1 & 4 \\
\hline \#\# & ehc28p3m14F & 4 & ehc28p3m14R & 3 & 1 & 4 \\
\hline \#\# & ehc28p3m15F & 4 & ehc28p3m15R & 3 & 1 & 4 \\
\hline \#\# & ehc $28 \mathrm{p} 3 \mathrm{~m} 16 \mathrm{~F}$ & 4 & ehc $28 p 3 m 16 R$ & 3 & 1 & 4 \\
\hline \#\# & ehc28p3m17F & 4 & ehc28p3m17R & 3 & 1 & 4 \\
\hline \#\# & ehc $16 \mathrm{~m} 4 \mathrm{~F}$ & 3 & ehc $16 \mathrm{~m} 4 \mathrm{R}$ & 3 & 2 & 308 \\
\hline \#\# & ehc16m5F & 3 & ehc16m5R & 3 & 2 & 660 \\
\hline \#\# & ehc16m6F & 3 & ehc16m6R & 3 & 4 & 1000 \\
\hline \#\# & ehc16m7F & 4 & ehc16m7R & 4 & 4 & 1412 \\
\hline \#\# & ehc16m8F & 4 & ehc16m8R & 4 & 4 & 1822 \\
\hline \#\# & ehc16m9F & 4 & ehc16m9R & 4 & 4 & 2276 \\
\hline \#\# & ehc16m10F & 4 & ehc16m10R & 4 & 4 & 2280 \\
\hline \#\# & ehc16m11F & 3 & ehc16m11R & 3 & 4 & 2280 \\
\hline \#\# & ehc16m12F & 3 & ehc16m12R & 3 & 4 & 2282 \\
\hline \#\# & ehc16m13F & 3 & ehc16m13R & 4 & 5 & 2281 \\
\hline \#\# & ehc16m14F & 4 & ehc16m14R & 4 & 6 & 2282 \\
\hline \#\# & ehc16m15F & 3 & ehc16m15R & 4 & 5 & 2280 \\
\hline \#\# & ehc16m16F & 3 & ehc16m16R & 4 & 5 & 2285 \\
\hline \#\# & ehc16m17F & 3 & ehc16m17R & 4 & 5 & 2284 \\
\hline \#\# & ehc16F & 3 & ehc16R & 4 & 5 & 2284 \\
\hline \#\# & ehc22p1nF & 5 & ehc22p1nR & 4 & 5 & 63 \\
\hline \#\# & ehc22p2nF & 4 & ehc $22 \mathrm{p} 2 \mathrm{nR}$ & 5 & 7 & 69 \\
\hline \#\# & ehc $22 p 3 n F$ & 4 & ehc22p3nR & 4 & 6 & 35 \\
\hline \#\# & ehc22p $4 n F$ & 4 & ehc $22 p 4 n R$ & 3 & 5 & 13 \\
\hline \#\# & ehc22p5nF & 3 & ehc22p5nR & 3 & 4 & 5 \\
\hline \#\# & ehc22p7nF & 2 & ehc22p $7 n R$ & 3 & 1 & 1 \\
\hline \#\# & ehc23p1F & 12 & ehc23p1R & 12 & 30 & 71 \\
\hline \#\# & ehc23p2F & 12 & ehc23p2R & 14 & 26 & 74 \\
\hline \#\# & ehc23p3F & 12 & ehc23p3R & 13 & 23 & 40 \\
\hline \#\# & ehc23p4F & 8 & ehc23p4R & 8 & 10 & 16 \\
\hline \#\# & ehc23p5F & 4 & ehc23p5R & 4 & 2 & 4 \\
\hline \#\# & ehc24p1mF & 15 & ehc $24 \mathrm{p} 1 \mathrm{mR}$ & 14 & 13 & 48 \\
\hline \#\# & ehc24p $2 m F$ & 15 & ehc $24 \mathrm{p} 2 \mathrm{mR}$ & 14 & 15 & 49 \\
\hline \#\# & ehc 24 p $3 m F$ & 14 & ehc $24 p 3 m R$ & 13 & 9 & 28 \\
\hline \#\# & ehc24p $4 \mathrm{mF}$ & 8 & ehc $24 \mathrm{p} 4 \mathrm{mR}$ & 8 & 4 & 10 \\
\hline \#\# & ehc24p5mF & 5 & ehc $24 \mathrm{p} 5 \mathrm{mR}$ & 5 & 2 & 2 \\
\hline \#\# & ehc24p $6 \mathrm{mF}$ & 4 & ehc24p $6 \mathrm{mR}$ & 3 & 1 & 1 \\
\hline \#\# & ehc24p7mF & 3 & ehc24p7mR & 2 & 1 & 1 \\
\hline \#\# & ehc24p1yF & 45 & ehc24p1yR & 44 & 13 & 62 \\
\hline \#\# & ehc24p2yF & 42 & ehc24p2yR & 43 & 11 & 64 \\
\hline \#\# & ehc24p3yF & 24 & ehc24p3yR & 24 & 8 & 32 \\
\hline \#\# & ehc24p4yF & 11 & ehc24p4yR & 11 & 4 & 12 \\
\hline \#\# & ehc24p5yF & 6 & ehc24p5yR & 6 & 2 & 2 \\
\hline \#\# & ehc24p6yF & 4 & ehc24p6yR & 3 & 1 & 1 \\
\hline \#\# & ehc24p7yF & 3 & ehc24p7yR & 2 & 1 & 1 \\
\hline \#\# & ehc25p1h1F & 5 & ehc25p1h1R & 4 & 5 & 68 \\
\hline \#\# & ehc $25 \mathrm{p} 2 \mathrm{~h} 1 \mathrm{~F}$ & 5 & ehc $25 p 2 h 1 R$ & 4 & 7 & 81 \\
\hline \#\# & ehc25p3h1F & 5 & ehc25p3h1R & 4 & 3 & 44 \\
\hline \#\# & ehc25p4h1F & 5 & ehc $25 p 4 h 1 R$ & 4 & 5 & 14 \\
\hline \#\# & ehc25p5h1F & 4 & ehc25p5h1R & 4 & 2 & 6 \\
\hline \#\# & ehc25p7h1F & 3 & ehc25p7h1R & 4 & 1 & 2 \\
\hline \#\# & ehc25p1h2F & 3 & ehc25p1h2R & 4 & 3 & 316 \\
\hline \#\# & ehc $25 \mathrm{p} 2 \mathrm{~h} 2 \mathrm{~F}$ & 3 & ehc $25 \mathrm{p} 2 \mathrm{~h} 2 \mathrm{R}$ & 4 & 3 & 286 \\
\hline \#\# & ehc25p3h2F & 2 & ehc $25 p 3 h 2 R$ & 4 & 2 & 114 \\
\hline \#\# & ehc $25 \mathrm{p} 4 \mathrm{~h} 2 \mathrm{~F}$ & 2 & ehc $25 p 4 h 2 R$ & 4 & 2 & 30 \\
\hline
\end{tabular}




\begin{tabular}{|c|c|c|c|c|c|c|}
\hline \#\# & ehc26h1F & 3 & ehc26h1R & 3 & 6 & 261 \\
\hline \#\# & ehc26h2F & 3 & ehc26h2R & 3 & 4 & 1796 \\
\hline \#\# & ehc10k1h1F & 3 & ehc10k1h1R & 3 & 6 & 188 \\
\hline \#\# & ehc10k2h1F & 3 & ehc10k2h1R & 3 & 4 & 75 \\
\hline \#\# & ehc10k3h1F & 3 & ehc10k3h1R & 3 & 4 & 35 \\
\hline \#\# & ehc10k4h1F & 3 & ehc10k4h1R & 3 & 4 & 12 \\
\hline \#\# & ehc10k5h1F & 2 & ehc10k5h1R & 3 & 3 & 6 \\
\hline \#\# & ehc10k6h1F & 2 & ehc10k6h1R & 3 & 1 & 6 \\
\hline \#\# & ehc10k7h1F & 2 & ehc10k7h1R & 3 & 1 & 2 \\
\hline \#\# & ehc10k1h2F & 3 & ehc10k1h2R & 3 & 4 & 1448 \\
\hline \#\# & ehc10k2h2F & 3 & ehc10k2h2R & 3 & 4 & 836 \\
\hline \#\# & ehc10k3h2F & 3 & ehc10k3h2R & 3 & 2 & 244 \\
\hline \#\# & ehc10k4h2F & 3 & ehc10k4h2R & 3 & 2 & 84 \\
\hline \#\# & hhnrF & 3 & hhnrR & 3 & 4 & 1177 \\
\hline \#\# & hh1nrF & 4 & hh1nrR & 5 & 9 & 1197 \\
\hline \#\# & hh2nrF & 4 & hh2nrR & 3 & 3 & 3 \\
\hline \#\# & hh1aF & 3 & hh1aR & 2 & 3 & 8 \\
\hline \#\# & $\mathrm{hh} 2 \mathrm{aF}$ & 3 & hh2aR & 2 & 3 & 5 \\
\hline \#\# & hh11F & 13 & hh11R & 13 & 6 & 9 \\
\hline \#\# & hh2lF & 12 & hh2lR & 11 & 5 & 4 \\
\hline \#\# & hh1t0F & 2 & hh1t0R & 2 & 4 & 4 \\
\hline \#\# & hh2tOF & 3 & hh2toR & 3 & 4 & 4 \\
\hline \#\# & $\mathrm{hpF}$ & 2 & $\mathrm{hpR}$ & 2 & 4 & 2 \\
\hline \#\# & hpnrF & 3 & hpnrR & 3 & 4 & 4 \\
\hline \#\# & hpnF & 2 & hpnR & 2 & 4 & 4 \\
\hline \#\# & $h p g F$ & 3 & hpgR & 3 & 6 & 5 \\
\hline \#\# & hpOF & 2 & hpOR & 2 & 2 & 3 \\
\hline \#\# & hpsepF & 2 & hpsepR & 2 & 4 & 2 \\
\hline \#\# & hpmtF & 2 & hpmtR & 2 & 2 & 1 \\
\hline \#\# & $\mathrm{hpmF}$ & 3 & hpmR & 3 & 4 & 4 \\
\hline \#\# & $\mathrm{hpcF}$ & 3 & hpcR & 3 & 2 & 4 \\
\hline \#\# & hps $2 F$ & 2 & $\mathrm{hps} 2 \mathrm{R}$ & 2 & 4 & 2 \\
\hline \#\# & hp0nrF & 2 & hp0nrR & 2 & 2 & 2 \\
\hline \#\# & hpOnF & 2 & hpOnR & 2 & 4 & 4 \\
\hline \#\# & hp0gF & 3 & hp0gR & 3 & 2 & 2 \\
\hline \#\# & hpOctOF & 3 & hp0ctoR & 3 & 4 & 4 \\
\hline \#\# & hc6h1F & 7 & hc6h1R & 6 & 1 & 2 \\
\hline \#\# & hc $6 h 2 F$ & 8 & hc6h2R & 7 & 1 & 1 \\
\hline \#\# & job17F & 9 & job17R & 8 & 1 & 2 \\
\hline \#\# & job18hF & 6 & job18hR & 5 & 1 & 2 \\
\hline \#\# & sat $2 F$ & 14 & sat $2 \mathrm{R}$ & 13 & 3 & 2 \\
\hline \#\# & $\sin 3 i 1 F$ & 8 & $\sin 3 i 1 R$ & 7 & 3 & 2 \\
\hline \#\# & sin3i2F & 8 & sin3i2R & 7 & 3 & 2 \\
\hline \#\# & $\sin 1 \mathrm{~F}$ & 6 & $\sin 1 R$ & 5 & 3 & 3 \\
\hline \#\# & $\sin 2 F$ & 6 & $\sin 2 R$ & 5 & 3 & 3 \\
\hline \#\# & $\sin 6 i 1 F$ & 9 & sin6i1R & 8 & 1 & 1 \\
\hline \#\# & $\sin 6 i 2 F$ & 9 & $\sin 6 i 2 R$ & 8 & 1 & 1 \\
\hline \#\# & $\sin 6 i 3 F$ & 9 & sin6i3R & 8 & 1 & 2 \\
\hline \#\# & $\sin 6 i 4 F$ & 9 & sin6i4R & 8 & 1 & 2 \\
\hline \#\# & sin6i5F & 9 & sin6i5R & 8 & 1 & 2 \\
\hline \#\# & pa $2 \mathrm{mF}$ & 19 & pa $2 \mathrm{mR}$ & 18 & 1 & 2 \\
\hline \#\# & pa30F & 3 & pa30R & 3 & 2 & 2 \\
\hline \#\# & pa3F & 11 & pa3R & 10 & 1 & 1 \\
\hline \#\# & sdp1dF & 5 & sdp1dR & 4 & 1 & 2 \\
\hline \#\# & sdp $1 \mathrm{mF}$ & 16 & $\mathrm{sdp} 1 \mathrm{mR}$ & 15 & 3 & 15 \\
\hline \#\# & sdp1yF & 29 & sdp1yR & 28 & 3 & 23 \\
\hline
\end{tabular}




\begin{tabular}{|c|c|c|c|c|c|c|}
\hline \#\# & sdp2i1F & 4 & sdp2i1R & 3 & 1 & 2 \\
\hline \#\# & sdp2i2F & 3 & sdp2i2R & 2 & 1 & 2 \\
\hline \#\# & sdp2i3F & 3 & sdp2i3R & 2 & 1 & 2 \\
\hline \#\# & sdp2i $4 F$ & 3 & sdp2i4R & 2 & 1 & 2 \\
\hline \#\# & sdp2i5F & 3 & sdp2i5R & 2 & 1 & 2 \\
\hline \#\# & sdp2i6F & 3 & sdp2i6R & 2 & 1 & 2 \\
\hline \#\# & sdp2i7F & 3 & sdp2i7R & 2 & 1 & 2 \\
\hline \#\# & sdp2i8F & 3 & sdp2i8R & 2 & 1 & 2 \\
\hline \#\# & sdp2i9F & 3 & sdp2i9R & 2 & 1 & 2 \\
\hline \#\# & sdp2i10F & 3 & sdp2i10R & 2 & 1 & 2 \\
\hline \#\# & sdp2i11F & 3 & sdp2i11R & 2 & 1 & 2 \\
\hline \#\# & sdp2i12F & 4 & sdp2i12R & 3 & 1 & 2 \\
\hline \#\# & sdp3F & 3 & sdp3R & 2 & 1 & 2 \\
\hline \#\# & sdp6F & 5 & sdp6R & 4 & 1 & 2 \\
\hline \#\# & sdp21F & 7 & sdp21R & 6 & 1 & 2 \\
\hline \#\# & sdp22F & 18 & sdp22R & 18 & 4 & 2 \\
\hline \#\# & sdp10i16F & 6 & sdp10i16R & 6 & 4 & 2 \\
\hline \#\# & sdp10i17F & 6 & sdp10i17R & 6 & 4 & 2 \\
\hline \#\# & sdp10i7F & 6 & sdp10i7R & 6 & 4 & 2 \\
\hline \#\# & sdp10i18F & 6 & sdp10i18R & 6 & 4 & 2 \\
\hline \#\# & sdp10i19F & 6 & sdp10i19R & 6 & 4 & 2 \\
\hline \#\# & sdp10i9F & 6 & sdp10i9R & 6 & 4 & 2 \\
\hline \#\# & sdp10i20F & 6 & sdp10i20R & 6 & 4 & 2 \\
\hline \#\# & sdp10i21F & 6 & sdp10i21R & 6 & 4 & 2 \\
\hline \#\# & sdp10i22F & 5 & sdp10i22R & 5 & 4 & 2 \\
\hline \#\# & sdp10i1F & 6 & sdp10i1R & 6 & 4 & 2 \\
\hline \#\# & sdp10i2F & 6 & sdp10i2R & 6 & 4 & 2 \\
\hline \#\# & sdp10i3F & 6 & sdp10i3R & 6 & 4 & 2 \\
\hline \#\# & sdp10i4F & 6 & sdp10i4R & 6 & 4 & 2 \\
\hline \#\# & sdp10i5F & 6 & sdp10i5R & 6 & 4 & 2 \\
\hline \#\# & sdp10i6F & 6 & sdp10i6R & 6 & 4 & 2 \\
\hline \#\# & sdp10i13F & 6 & sdp10i13R & 6 & 4 & 2 \\
\hline \#\# & sdp10i8F & 6 & sdp10i8R & 6 & 4 & 2 \\
\hline \#\# & sdp10i10F & 5 & sdp10i10R & 5 & 4 & 2 \\
\hline \#\# & sdp10i11F & 6 & sdp10i11R & 6 & 4 & 2 \\
\hline \#\# & sdp10i12F & 6 & sdp10i12R & 6 & 4 & 2 \\
\hline \#\# & sdp10i15F & 6 & sdp10i15R & 6 & 4 & 2 \\
\hline \#\# & sdp10i14F & 6 & sdp10i14R & 6 & 4 & 2 \\
\hline \#\# & sdp $16 F$ & 5 & sdp16R & 4 & 3 & 3 \\
\hline \#\# & sdp17F & 5 & sdp17R & 4 & 1 & 2 \\
\hline \#\# & sdp18F & 6 & sdp18R & 6 & 4 & 2 \\
\hline \#\# & hcp1i1F & 5 & hcp1i1R & 6 & 1 & 2 \\
\hline \#\# & hcp1i2F & 17 & hcp1i2R & 18 & 1 & 2 \\
\hline \#\# & hcp2F & 8 & hcp2R & 9 & 1 & 2 \\
\hline \#\# & hcp $3 h F$ & 15 & hcp3hR & 16 & 1 & 2 \\
\hline \#\# & hcp $3 m$ F & 18 & hcp $3 m R$ & 19 & 1 & 2 \\
\hline \#\# & hcp 4 hF & 5 & hcp $4 h R$ & 4 & 1 & 2 \\
\hline \#\# & hcp5F & 6 & hcp5R & 6 & 4 & 2 \\
\hline \#\# & pa9F & 7 & pa9R & 8 & 1 & 2 \\
\hline \#\# & pa11F & 9 & pa11R & 8 & 1 & 1 \\
\hline \#\# & pa13F & 5 & pa13R & 5 & 2 & 1 \\
\hline \#\# & pa14i1F & 9 & pa14i1R & 9 & 2 & 2 \\
\hline \#\# & pa14i2F & 8 & pa14i2R & 8 & 2 & 2 \\
\hline \#\# & pa14i3F & 10 & pa14i3R & 10 & 2 & 2 \\
\hline \#\# & pa14i4F & 9 & pa14i4R & 9 & 2 & 2 \\
\hline \#\# & pa14i5F & 8 & pa14i5R & 7 & 1 & 1 \\
\hline
\end{tabular}




\begin{tabular}{|c|c|c|c|c|c|c|}
\hline \#\# & pa15F & 9 & pa15R & 9 & 2 & 2 \\
\hline \#\# & $\mathrm{cps} 1 \mathrm{~F}$ & 4 & $\operatorname{cps} 1 \mathrm{R}$ & 4 & 4 & 2 \\
\hline \#\# & sat3F & 14 & sat3R & 14 & 4 & 2 \\
\hline \#\# & pa26F & 6 & pa26R & 6 & 4 & 2 \\
\hline \#\# & pa27F & 6 & pa27R & 6 & 4 & 2 \\
\hline \#\# & pa28F & 6 & pa28R & 6 & 4 & 2 \\
\hline \#\# & sep1i1F & 8 & sep1i1R & 8 & 2 & 1 \\
\hline \#\# & sep1i2F & 8 & sep1i2R & 8 & 2 & 1 \\
\hline \#\# & sep2F & 7 & sep2R & 7 & 2 & 1 \\
\hline \#\# & sep18F & 5 & sep18R & 5 & 2 & 1 \\
\hline \#\# & sep12F & 6 & sep12R & 6 & 2 & 1 \\
\hline \#\# & sep13F & 4 & sep13R & 4 & 2 & 1 \\
\hline \#\# & sep14F & 6 & sep14R & 6 & 2 & 1 \\
\hline \#\# & sep17i1F & 7 & sep17i1R & 6 & 3 & 3 \\
\hline \#\# & sep17i2F & 7 & sep17i2R & 6 & 3 & 3 \\
\hline \#\# & sep17i3F & 7 & sep17i3R & 6 & 3 & 3 \\
\hline \#\# & sep17i4F & 7 & sep17i4R & 6 & 3 & 3 \\
\hline \#\# & sep16F & 6 & sep16R & 6 & 2 & 3 \\
\hline \#\# & frt $2 F$ & 8 & frt $2 R$ & 8 & 4 & 2 \\
\hline \#\# & frt11v1i6F & 10 & frt11v1i6R & 10 & 4 & 2 \\
\hline \#\# & frt11v1i7F & 9 & frt11v1i7R & 9 & 4 & 2 \\
\hline \#\# & frt11v1i8F & 9 & frt11v1i8R & 9 & 4 & 2 \\
\hline \#\# & frt11v2i6F & 6 & frt11v2i6R & 6 & 4 & 2 \\
\hline \#\# & frt11v2i7F & 6 & frt11v2i7R & 6 & 4 & 2 \\
\hline \#\# & frt11v2i8F & 6 & frt11v2i8R & 6 & 4 & 2 \\
\hline \#\# & crn22p1F & 11 & crn22p1R & 11 & 2 & 2 \\
\hline \#\# & crn33p1i1F & 7 & crn33p1i1R & 7 & 2 & 2 \\
\hline \#\# & crn33p1i2F & 6 & crn33p1i2R & 5 & 1 & 2 \\
\hline \#\# & crn33p1i3F & 8 & crn33p1i3R & 8 & 2 & 2 \\
\hline \#\# & crn34p1F & 7 & crn34p1R & 7 & 2 & 2 \\
\hline \#\# & igr $22 \mathrm{~F}$ & 5 & igr $22 \mathrm{R}$ & 4 & 1 & 14 \\
\hline \#\# & igr $25 \mathrm{~F}$ & 5 & igr25R & 4 & 3 & 13 \\
\hline \#\# & igr $28 \mathrm{~F}$ & 5 & igr28R & 4 & 1 & 2 \\
\hline \#\# & igr39p3F & 10 & igr39p3R & 9 & 1 & 2 \\
\hline \#\# & igr 40p3F & 9 & igr40p3R & 8 & 1 & 1 \\
\hline \#\# & igr $41 \mathrm{p} 1 \mathrm{~F}$ & 10 & igr $41 \mathrm{p} 1 \mathrm{R}$ & 9 & 5 & 14 \\
\hline \#\# & $\operatorname{rtr} 43 \mathrm{~F}$ & 5 & rtr $43 \mathrm{R}$ & 5 & 2 & 1 \\
\hline \#\# & inc $24 \mathrm{~F}$ & 38 & inc $24 \mathrm{R}$ & 37 & 1 & 7 \\
\hline \#\# & inc26i2F & 7 & inc26i2R & 6 & 11 & 20 \\
\hline \#\# & inc $26 i 3 F$ & 7 & inc26i3R & 6 & 9 & 20 \\
\hline \#\# & inc27i2F & 8 & inc27i2R & 7 & 1 & 9 \\
\hline \#\# & inc27i3F & 8 & inc27i3R & 7 & 1 & 9 \\
\hline \#\# & int4i6F & 4 & int4i6R & 4 & 4 & 2 \\
\hline \#\# & $\operatorname{lmhh1F}$ & 2 & $\operatorname{lmhh1R}$ & 2 & 2 & 4 \\
\hline \#\# & $\operatorname{lmhh} 2 \mathrm{~F}$ & 2 & $\operatorname{lmhh} 2 \mathrm{R}$ & 2 & 2 & 3 \\
\hline \#\# & Ivhh1F & 2 & Ivhh1R & 2 & 4 & 4 \\
\hline \#\# & lvhh2F & 2 & lvhh2R & 2 & 2 & 3 \\
\hline \#\# & $\operatorname{avhh1F}$ & 2 & avhh1R & 2 & 2 & 1 \\
\hline \#\# & hv1F & 4 & hv1R & 3 & 1 & 2 \\
\hline \#\# & tag_sexF & 2 & tag_sexR & 2 & 2 & 1 \\
\hline \#\# & tag_dobpF & 2 & tag_dobpR & 2 & 4 & 25 \\
\hline \#\# & sex_genF & 2 & sex_genR & 2 & 2 & 1 \\
\hline \#\# & psex_genF & 3 & psex_genR & 4 & 5 & 5 \\
\hline \#\# & pdoby_genF & 43 & pdoby_genR & 44 & 15 & 15 \\
\hline \#\# & pdobm_genF & 14 & pdobm_genR & 15 & 9 & 9 \\
\hline \#\# & pageF & 44 & pageR & 44 & 16 & 11 \\
\hline
\end{tabular}




\begin{tabular}{|c|c|c|c|c|c|c|}
\hline \#\# & mageF & 40 & mageR & 40 & 4 & 3 \\
\hline \#\# & k1ageF & 28 & k1ageR & 28 & 2 & 1 \\
\hline \#\# & $\mathrm{k} 2 \mathrm{ageF}$ & 26 & k2ageR & 26 & 4 & 2 \\
\hline \#\# & pcobF & 18 & pcobR & 19 & 3 & 4 \\
\hline \#\# & pnat1F & 15 & pnat1R & 16 & 3 & 3 \\
\hline \#\# & marstatF & 5 & marstatR & 5 & 2 & 1 \\
\hline \#\# & $\mathrm{npF}$ & 10 & $n p R$ & 10 & 20 & 23 \\
\hline \#\# & ncohF & 7 & ncohR & 7 & 10 & 17 \\
\hline \#\# & $\mathrm{nmarF}$ & 3 & nmarR & 3 & 6 & 12 \\
\hline \#\# & meetdurF & 279 & meetdurR & 278 & 13 & 18 \\
\hline \#\# & reldurF & 265 & reldurR & 262 & 37 & 94 \\
\hline \#\# & cohabdurF & 242 & cohabdurR & 249 & 187 & 134 \\
\hline \#\# & mardurF & 191 & mardurR & 192 & 13 & 14 \\
\hline \#\# & homosexF & 3 & homosexR & 3 & 4 & 2 \\
\hline \#\# & infertileF & 3 & infertileR & 3 & 4 & 4 \\
\hline \#\# & pregnantF & 4 & pregnantR & 4 & 6 & 4 \\
\hline \#\# & pnkidsbioalvF & 7 & pnkidsbioalvR & 7 & 12 & 64 \\
\hline \#\# & res $2 \mathrm{ndF}$ & 3 & res $2 \mathrm{ndR}$ & 2 & 3 & 1175 \\
\hline \#\# & hhsizemrdF & 13 & hhsizemrdR & 13 & 16 & 17 \\
\hline \#\# & $\operatorname{mmrdF}$ & 2 & $\operatorname{mmrdR}$ & 2 & 2 & 4 \\
\hline \#\# & $f m r d F$ & 2 & $f m r d R$ & 3 & 3 & 4 \\
\hline \#\# & childmrdF & 9 & childmrdR & 8 & 7 & 8 \\
\hline \#\# & othmrdF & 5 & othmrdR & 5 & 2 & 1 \\
\hline \#\# & hhcompF & 14 & hhcompR & 15 & 21 & 29 \\
\hline \#\# & penrolf & 11 & penrolR & 11 & 2 & 1 \\
\hline \#\# & pschoolf & 10 & pschoolR & 10 & 6 & 5 \\
\hline \#\# & pvocatF & 11 & pvocatR & 11 & 4 & 2 \\
\hline \#\# & piscedF & 11 & piscedR & 11 & 6 & 3 \\
\hline \#\# & pisced2F & 11 & pisced2R & 11 & 6 & 3 \\
\hline \#\# & pcasminf & 13 & pcasminR & 13 & 4 & 2 \\
\hline \#\# & pyeducF & 20 & pyeducR & 20 & 6 & 3 \\
\hline \#\# & egpF & 129 & egpR & 11 & 264 & 3514 \\
\hline \#\# & iseiF & 73 & iseiR & 56 & 451 & 1720 \\
\hline \#\# & siopsF & 55 & siopsR & 54 & 431 & 1331 \\
\hline \#\# & hhincgceeF & 413 & hhincgceeR & 414 & 21 & 118 \\
\hline \#\# & pcasprimF & 22 & pcasprimR & 22 & 4 & 3 \\
\hline \#\# & pcassecF & 17 & pcassecR & 17 & 2 & 1 \\
\hline \#\# & plfsF & 14 & plfsR & 14 & 4 & 3 \\
\hline
\end{tabular}

\section{B.3. variation and bijective mapping}

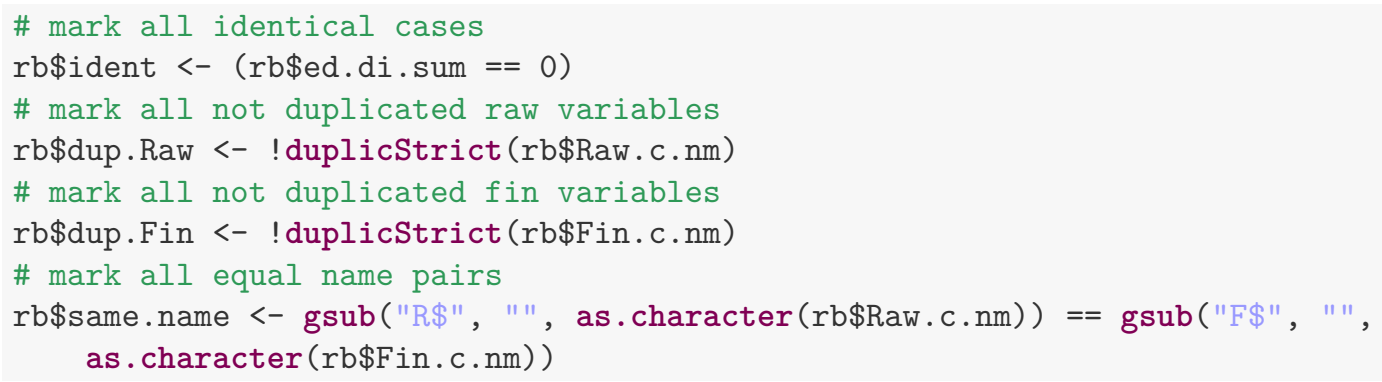


rb\$Raw.ident <- (ave(rb\$ident, rb\$Raw.c.nm, FUN = sum) >0)

rb\$Fin.ident <- (ave (rb\$ident, rb\$Fin.c.nm, FUN = sum) >0)

R.vb <- length (unique (rb[!rb\$dup.Raw \& !rb\$Raw.same.name \& !rb\$Raw.ident, "Raw.c.nm"])) as. character (unique (rb[!rb\$dup. Raw \& !rb\$Raw.same.name \& !rb\$Raw.ident, "Raw.c.nm"]))

\#\# [1] "ehc24p7yR" "ehc25p7h1R"

R.vbi <- length (unique(rb[!rb\$dup.Raw \& !rb\$Raw.same.name \& rb\$Raw.ident, "Raw.c.nm"])) as.character (unique (rb[!rb\$dup. Raw \& !rb\$Raw.same.name \& rb\$Raw.ident, "Raw.c.nm"]))

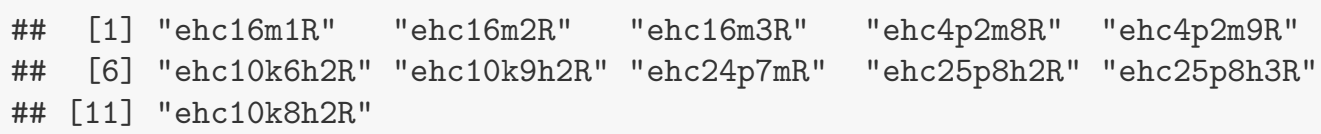

R.vbn <- length (unique (rb[!rb\$dup.Raw \& rb\$Raw.same.name \& !rb\$Raw.ident, "Raw.c.nm"])) as.character (unique (rb[!rb\$dup. Raw \& rb\$Raw.same.name \& !rb\$Raw.ident, "Raw.c.nm"]))

\#\# [1] "dpidR" "hcp4mR"

(R.vbni <- length(unique(rb[!rb\$dup.Raw \& rb\$Raw.same.name \& rb\$Raw.ident, "Raw.c.nm"])))

\#\# [1] 954

R.vb1 <- length (unique (rb[rb\$dup.Raw \& !rb\$same.name \& !rb\$ident, "Raw.c.nm"])) as.character (unique (rb[rb\$dup. Raw \& !rb\$same.name \& !rb\$ident, "Raw.c.nm"]))

\#\# [1] "ehc17m4R" "ehc22p7nR" "ehc25p6h2R" "ehc25p6h3R"

R.vb1i <- length (unique (rb[rb\$dup. Raw \& !rb\$same.name \& rb\$ident, "Raw.c.nm"])) as.character (unique (rb[rb\$dup. Raw \& !rb\$same.name \& rb\$ident, "Raw.c.nm"]))

\#\# [1] "ehc10k5h2R" "ehc10k7h2R" "kldbR"

R.vb1n <- length (unique (rb[rb\$dup.Raw \& rb\$same.name \& !rb\$ident, "Raw.c.nm"])) as.character (unique (rb[rb\$dup. Raw \& rb\$same.name \& !rb\$ident, "Raw.c.nm"]))

\#\# [1] "ehc21h1R" "job18mR" "pa2yR"

(R.vb1ni <- length(unique(rb[rb\$dup.Raw \& rb\$same.name \& rb\$ident, "Raw.c.nm"])))

\#\# [1] 1262

(F.vb <- length (unique(rb[!rb\$dup.Fin \& !rb\$Fin.same.name \& !rb\$Fin.ident, "Fin.c.nm"])))

\#\# [1] 0

F.vbi <- length(unique(rb[!rb\$dup.Fin \& !rb\$Fin.same.name \& rb\$Fin.ident, "Fin.c.nm"])) as.character (unique(rb[!rb\$dup.Fin \& !rb\$Fin.same.name \& rb\$Fin.ident, "Fin.c.nm"])) 


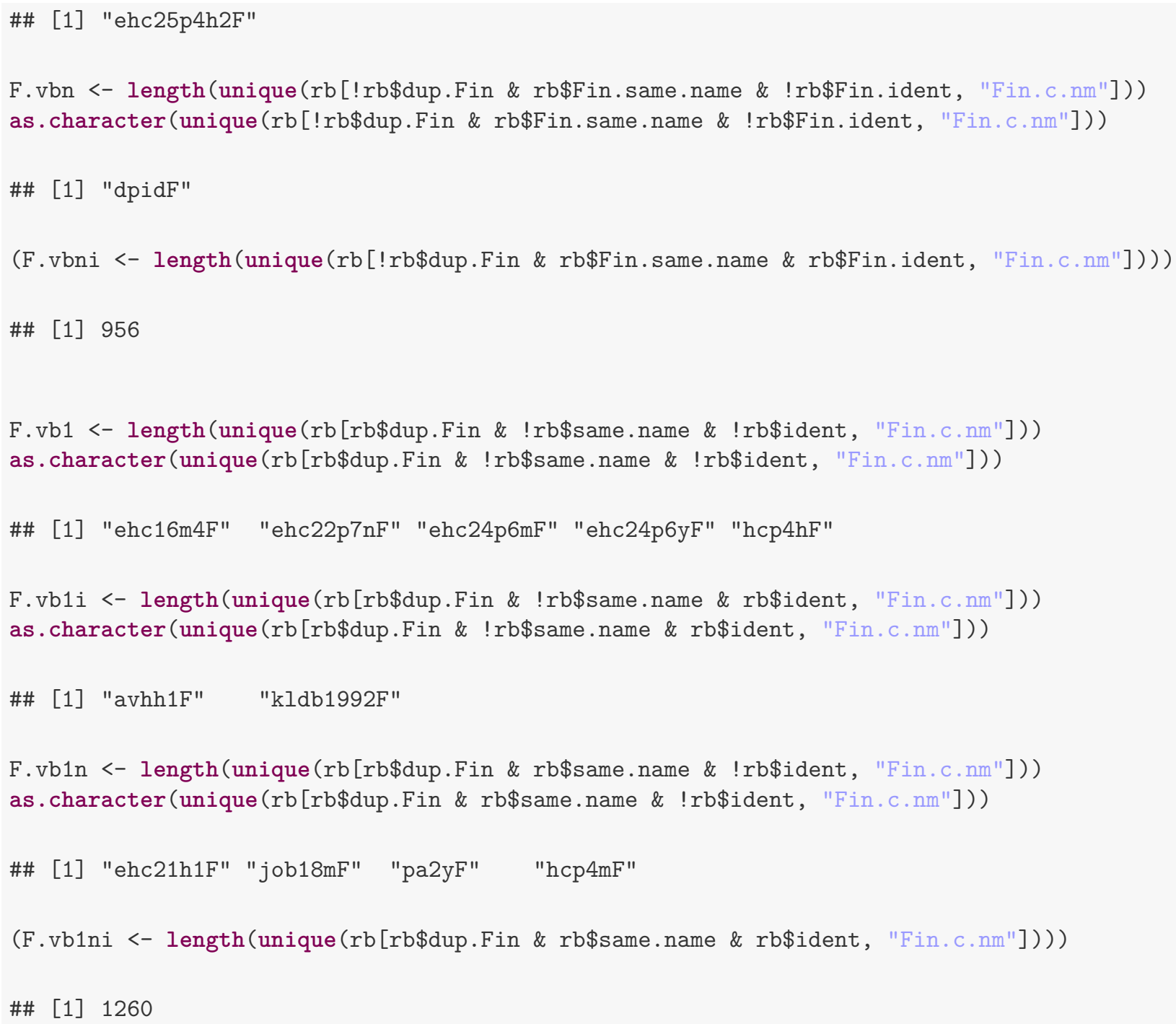

\section{B.4. comparison summary for anchor2_DD.dta Release 2.0 and Release 3.0}

\begin{tabular}{rrr} 
class & Release 2 & Release 3 \\
\hline $\mathrm{x}$ & 8 & 0 \\
$\mathrm{n}$ & 15 & 37 \\
$\mathrm{ni}$ & 612 & 612 \\
\hline $\mathrm{v}$ & 1 & 7 \\
$\mathrm{vn}$ & 369 & 359 \\
\hline $\mathrm{vb}$ & 2 & 0 \\
$\mathrm{vbi}$ & 11 & 1 \\
vbn & 2 & 1 \\
vbni & 954 & 956 \\
vb1 & 4 & 5 \\
vb1i & 3 & 2 \\
vb1n & 3 & 4 \\
vb1ni & 1262 & 1260 \\
\hline sum & 3246 & 3244
\end{tabular}

Most variables are unchanged (ni, vbni, vb1ni). A small number (x, v) had been dropped or added. Some variables (n) had lost or gained variation or changed to another constant value. A number of variables (vb, 
vbi, vbn, vb1, vb1i, vb1n) had been (probably) renamed or recoded. A huge group of variables (vn) had been changed.

\section{B.5. selected in-depth comparison}

For illustrative purposes we present some detailed results.

D1 <- cbind (Raw\$pyeducR, Fin\$pyeducF)

$\mathrm{D} 1[\mathrm{D} 1[, 1]$ ! = D1 $[, 2]$,

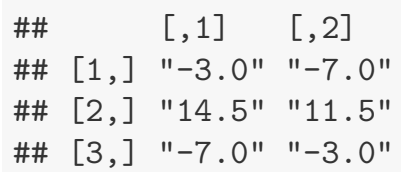

Both variables share the same number of levels. The level '-3.0' has been recoded to '-7.0', the level '14.5' to '11.5' and the level '-7.0' to '-30'. This takes 3 Levenshtein steps (see details in [TR-2012-003]).

D2 <- cbind (Raw\$nmarR, Fin\$nmarF)

$\mathrm{D} 2[\mathrm{D} 2[, 1] \quad !=\mathrm{D} 2[, 2]$,

$\begin{array}{lrrrr}\text { \#\# } & & {[, 1]} & {[, 2]} \\ \text { \#\# } & {[1,]} & \text { "0" } & \text { "1" } \\ \text { \#\# } & {[2,]} & \text { "0" } & \text { "1" } \\ \text { \#\# } & {[3,]} & \text { "0" } & \text { "1" } \\ \text { \#\# } & {[4,]} & \text { "0" } & \text { "1" } \\ \text { \#\# } & {[5,]} & \text { "0" } & \text { "1" } \\ \text { \#\# } & {[6,]} & \text { "1" } & \text { "0" } \\ \text { \#\# } & {[7,]} & \text { "1" } & \text { "0" } \\ \text { \#\# } & {[8,]} & \text { "0" } & \text { "1" } \\ \text { \#\# } & {[9,]} & \text { "0" } & \text { "1" } \\ \text { \#\# } & {[10,]} & \text { "0" } & \text { "1" } \\ \text { \#\# } & {[11,]} & \text { "0" } & \text { "1" } \\ \text { \#\# } & {[12,]} & \text { "1" } & \text { "2" }\end{array}$

Both variables share the same number of levels. $3+3+6=12,12 / 2=6$ mappings between the levels are in use. For a bijective mapping we need 3 mappings less. 12 individuals show a changed level. It takes 12 Levenshtein steps to make the levels equal.

D3 <- cbind (Raw\$pageR, Fin\$pageF)

D3 $[\mathrm{D} 3[, 1] \quad !=\mathrm{D} 3[, 2]$,

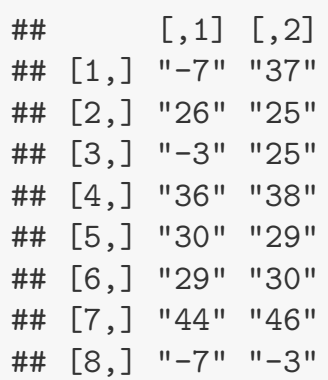

8 idividals have a new level for 'page'. It takes 11 Levenshtein steps to get them equal. There is no bijective mapping available. 
D4 <- cbind(Raw\$iseiR, Fin\$iseiF)

$\operatorname{dim}(\mathrm{D} 4[\mathrm{D} 4[, 1] \quad !=\mathrm{D} 4[, 2]]$,

\#\# [1] $1128 \quad 2$

$\operatorname{head}(\mathrm{D} 4[\mathrm{D} 4[, 1] \quad !=\mathrm{D} 4[, 2]]$,

\#\# [,1] [,2]

\#\# [1,] "23" "22"

\#\# [2,] "32" "33"

\#\# [3,] "53" "31"

\#\# [4,] "38" "56"

\#\# [5,] "48" "50"

\#\# [6,] "51" "48"

1128 indiduals do have a different level. There is no bijective mapping.

\section{C. partner1}

As usual we have first to load the produced data collection.

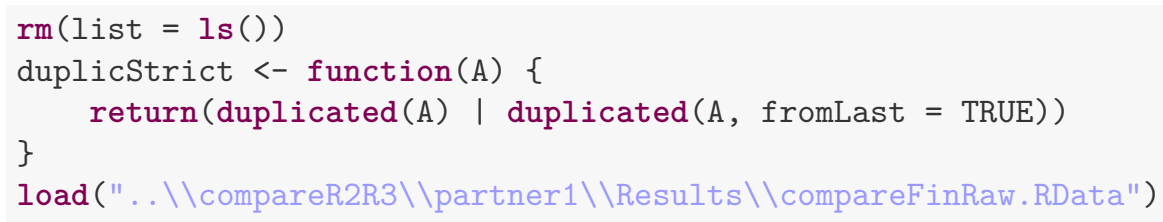

\section{C.1. no variation}

We compare the variables without variation first.

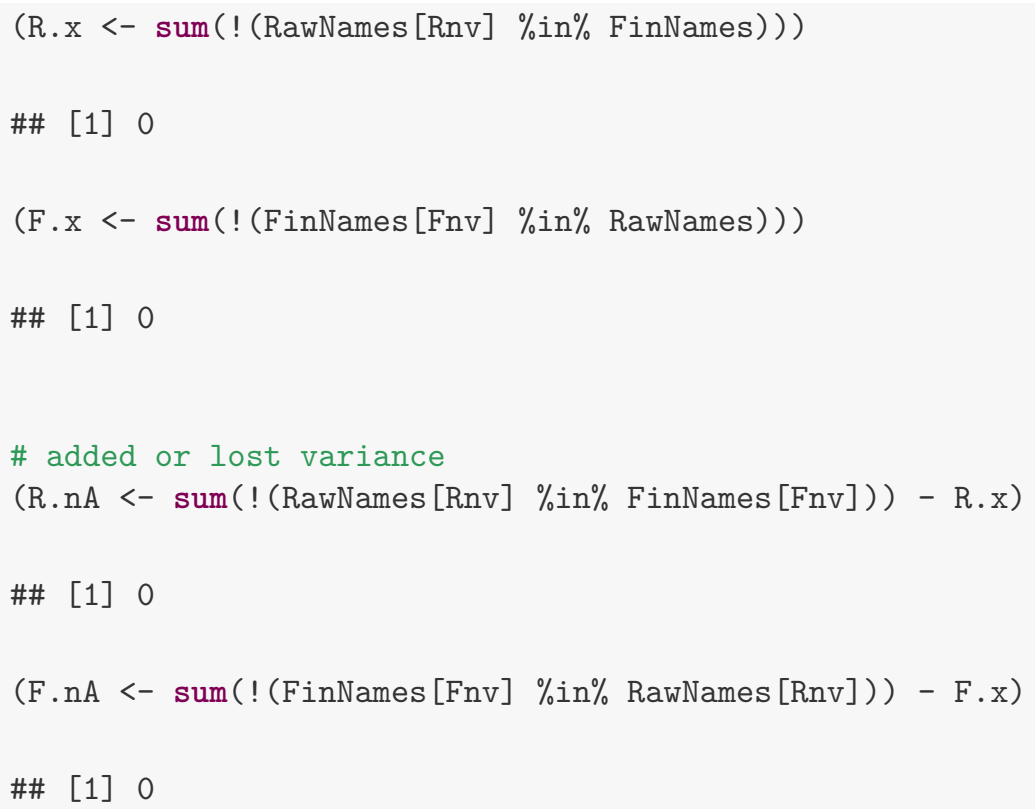




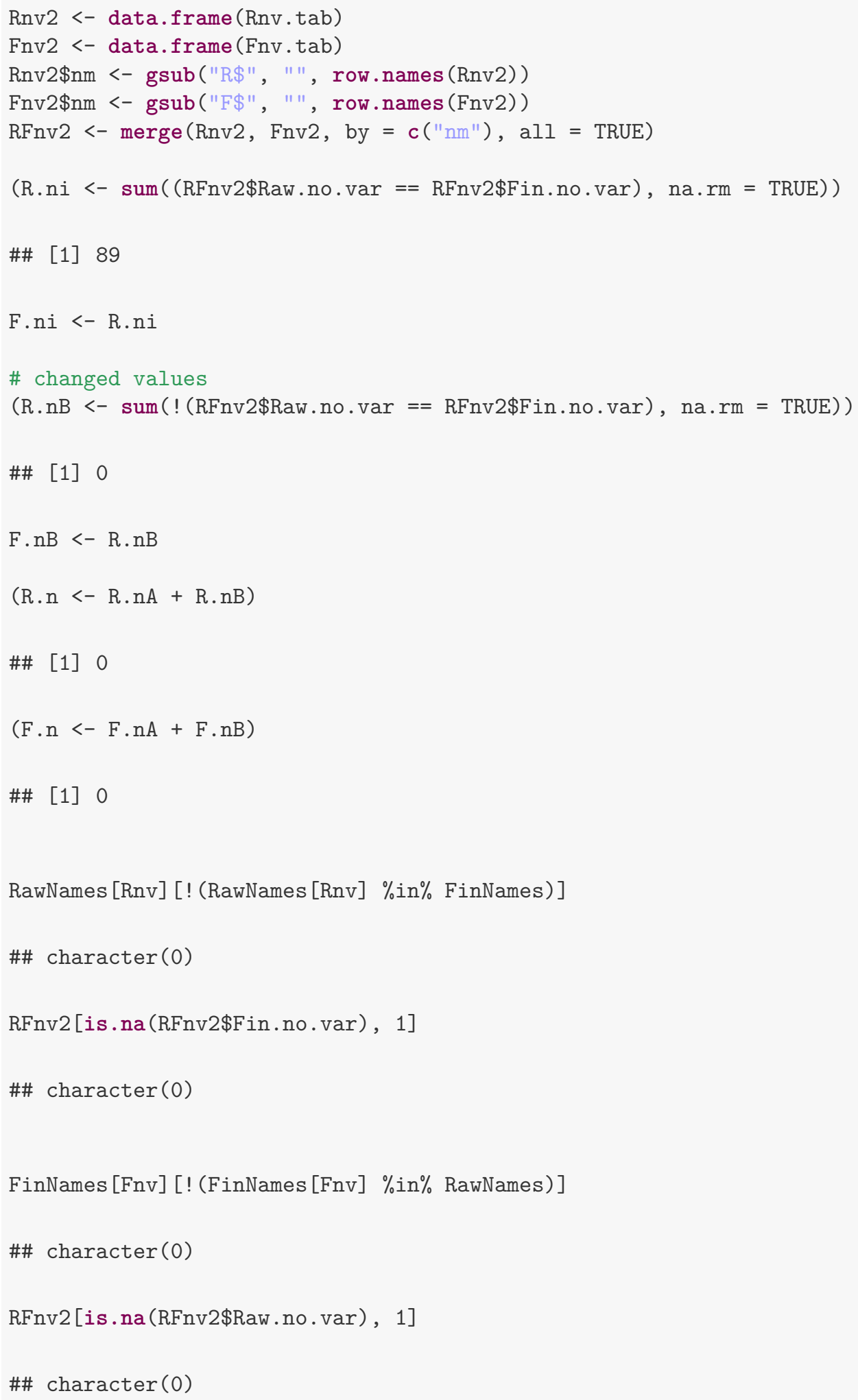

Release 2.0 89 variables without variation are identical in both data sets.

Release 3.0 89 variables without variation are identical in both data sets. 


\section{C.2. variation, but no bijective mapping}

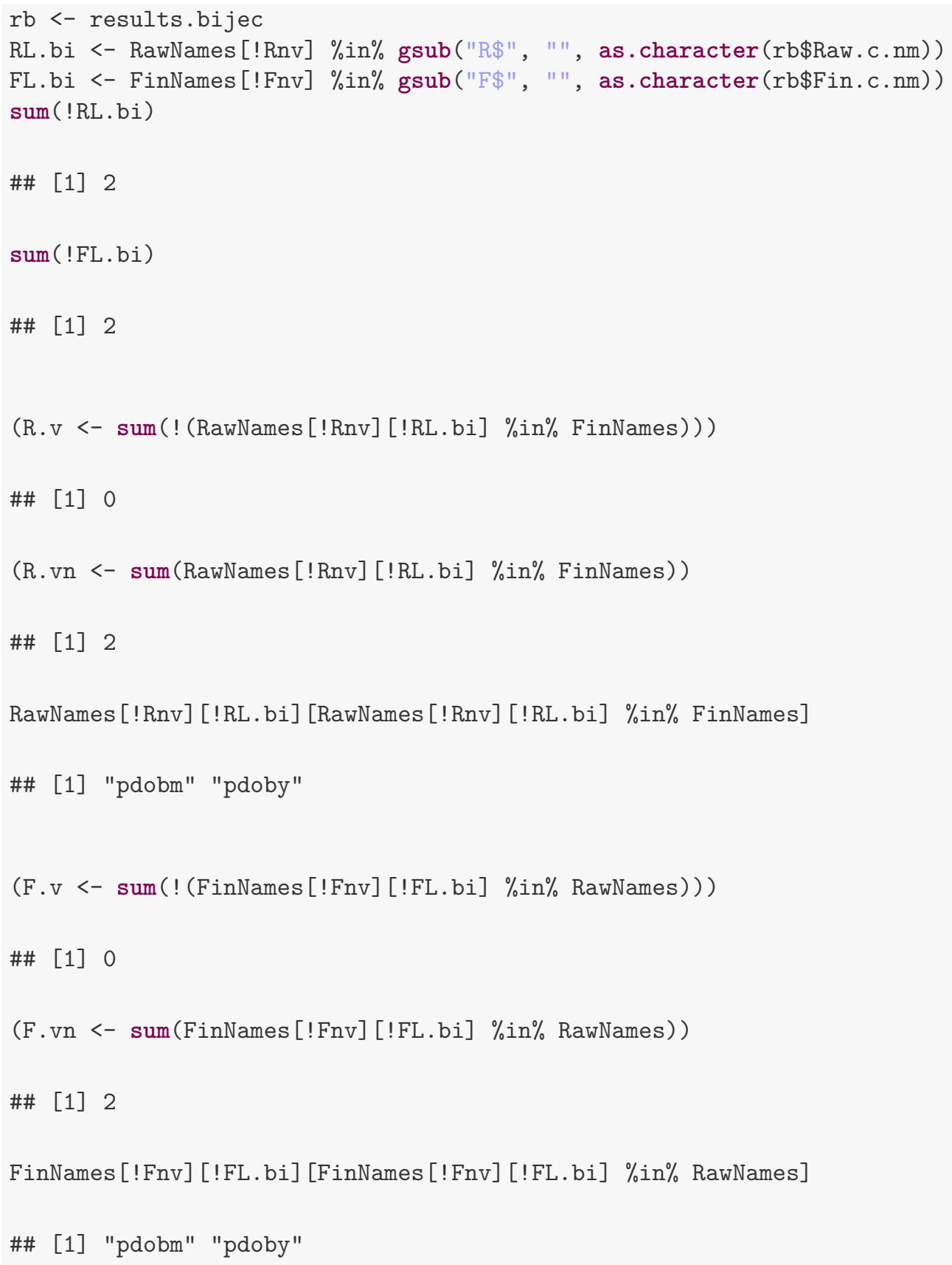

Release 2.0 2 variables share the name with one of the variables in Release 3.0 at least.

Release 3.0 2 variables share the name with one of the variables in Release 2.0 at least.

We compare all variables pairs (with variation) which share the same name but are not connected with a bijective mapping.

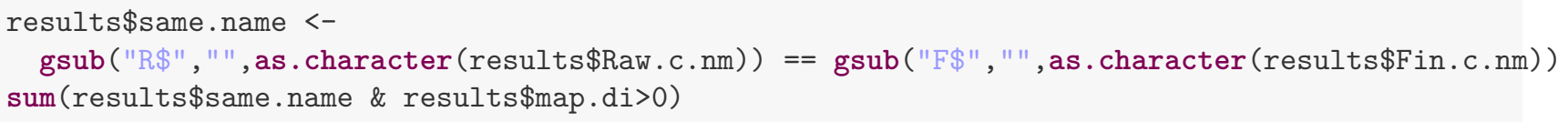


\#\# [1] 2

print(results [(results\$same.name \& results\$map.di>0),c(2,3,5,6,7,8)], row. names=FALSE)

\#\# Fin.c.nm Fin.c.ls Raw.c.nm Raw.c.ls map.di ed.di.sum

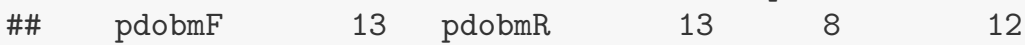

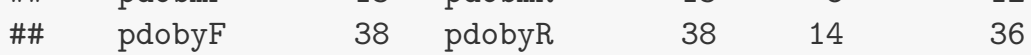

\section{C.3. variation and bijective mapping}

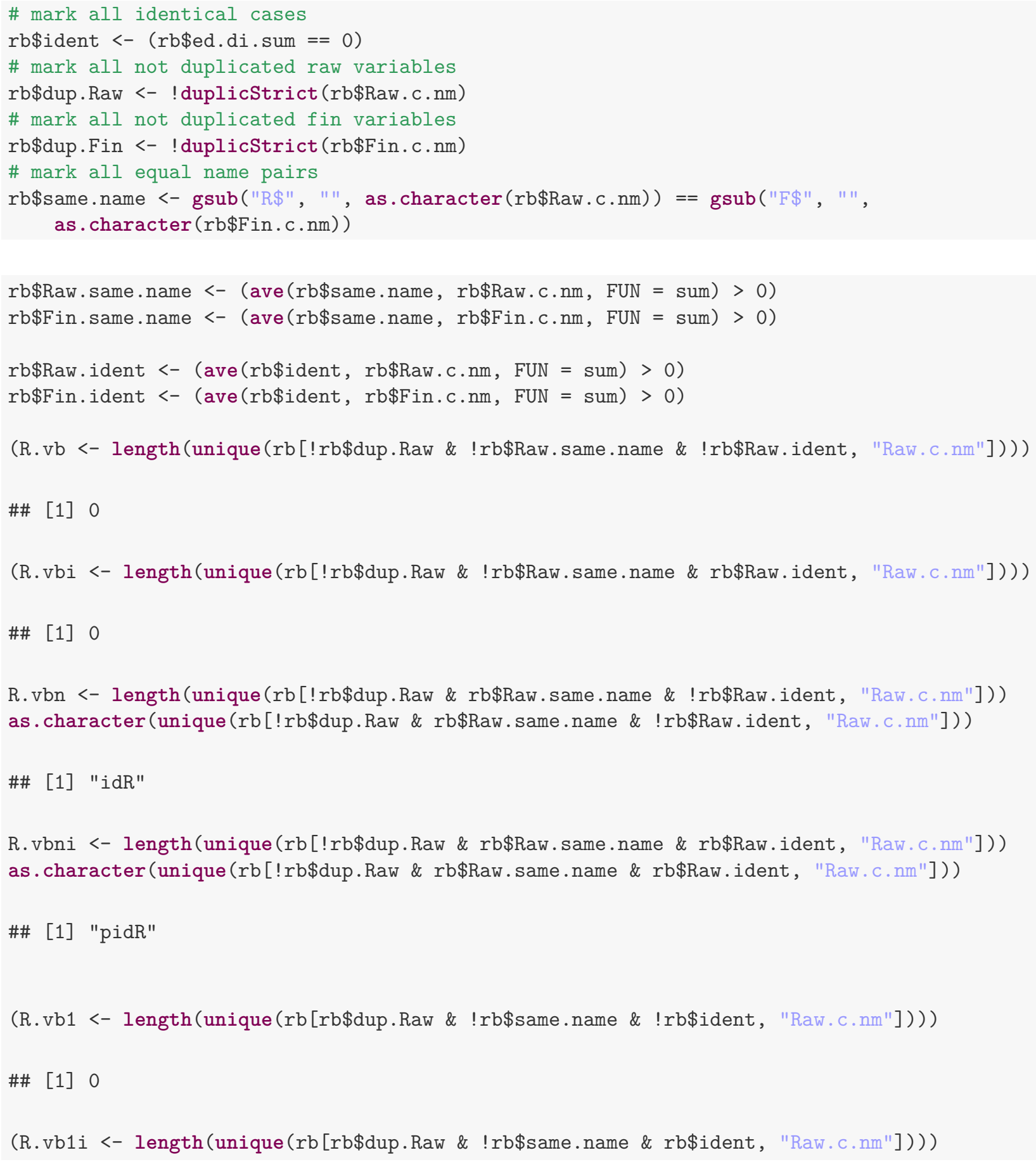




$$
\text { \#\# [1] } 0
$$

R.vb1n <- length (unique (rb[rb\$dup. Raw \& rb\$same.name \& !rb\$ident, "Raw.c.nm"])) as.character (unique (rb[rb\$dup. Raw \& rb\$same.name \& !rb\$ident, "Raw.c.nm"]))

\#\# [1] "psd10R"

(R.vb1ni <- length(unique(rb[rb\$dup.Raw \& rb\$same.name \& rb\$ident, "Raw.c.nm"])))

\#\# [1] 137

(F.vb <- length(unique(rb[!rb\$dup.Fin \& !rb\$Fin.same.name \& !rb\$Fin.ident, "Fin.c.nm"]))) \#\# [1] 0

(F.vbi <- length(unique(rb[!rb\$dup.Fin \& !rb\$Fin.same.name \& rb\$Fin.ident, "Fin.c.nm"]))) \#\# [1] 0

F.vbn <- length(unique(rb[!rb\$dup.Fin \& rb\$Fin.same.name \& !rb\$Fin.ident, "Fin.c.nm"])) as.character (unique (rb[!rb\$dup.Fin \& rb\$Fin.same.name \& !rb\$Fin.ident, "Fin.c.nm"]))

\#\# [1] "idF"

F.vbni <- length (unique(rb[!rb\$dup.Fin \& rb\$Fin.same.name \& rb\$Fin.ident, "Fin.c.nm"])) as.character (unique (rb[!rb\$dup.Fin \& rb\$Fin.same.name \& rb\$Fin.ident, "Fin.c.nm"]))

\#\# [1] "pidF"

(F.vb1 <- length(unique (rb[rb\$dup.Fin \& !rb\$same.name \& !rb\$ident, "Fin.c.nm"])))

\#\# [1] 0

(F.vb1i <- length(unique(rb[rb\$dup.Fin \& !rb\$same.name \& rb\$ident, "Fin.c.nm"])))

\#\# [1] 0

F.vb1n <- length (unique (rb[rb\$dup.Fin \& rb\$same.name \& !rb\$ident, "Fin.c.nm"])) as.character (unique (rb[rb\$dup.Fin \& rb\$same.name \& !rb\$ident, "Fin.c.nm"]))

\#\# [1] "psd10F"

(F.vb1ni <- length(unique(rb[rb\$dup.Fin \& rb\$same.name \& rb\$ident, "Fin.c.nm"])))

\#\# [1] 137 


\section{C.4. comparison summary for partner1_DD.dta Release 2.0 and Release 3.0}

\begin{tabular}{rrr} 
class & Release 2 & Release 3 \\
\hline $\mathrm{x}$ & 0 & 0 \\
$\mathrm{n}$ & 0 & 0 \\
$\mathrm{ni}$ & 89 & 89 \\
\hline $\mathrm{v}$ & 0 & 0 \\
$\mathrm{vn}$ & 2 & 2 \\
\hline $\mathrm{vb}$ & 0 & 0 \\
$\mathrm{vbi}$ & 0 & 0 \\
$\mathrm{vbn}$ & 1 & 1 \\
vbni & 1 & 1 \\
$\mathrm{vb1}$ & 0 & 0 \\
$\mathrm{vb} 1 \mathrm{i}$ & 0 & 0 \\
$\mathrm{vb1n}$ & 1 & 1 \\
vb1ni & 137 & 137 \\
\hline sum & 231 & 231
\end{tabular}

Most variables are unchanged (ni, vbni, vb1ni). A small number of variables (vbn, vb1n) had been (probably) renamed or recoded. A small group of variables (vn) had been changed.

\section{D. partner2}

As usual we have first to load the produced data collection.

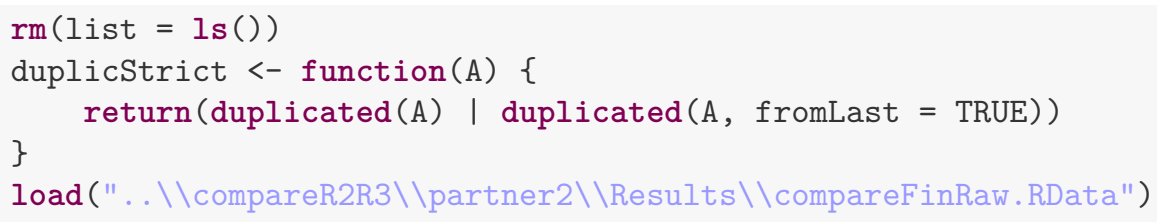

\section{D.1. no variation}

We compare the variables without variation first.

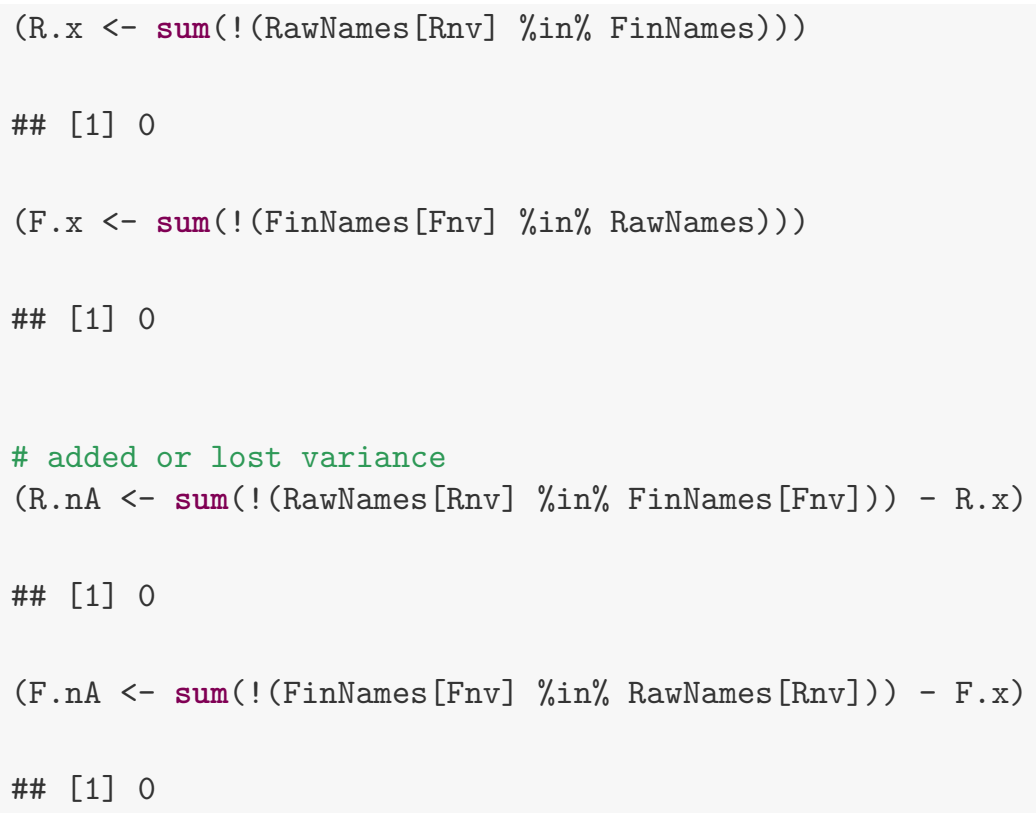




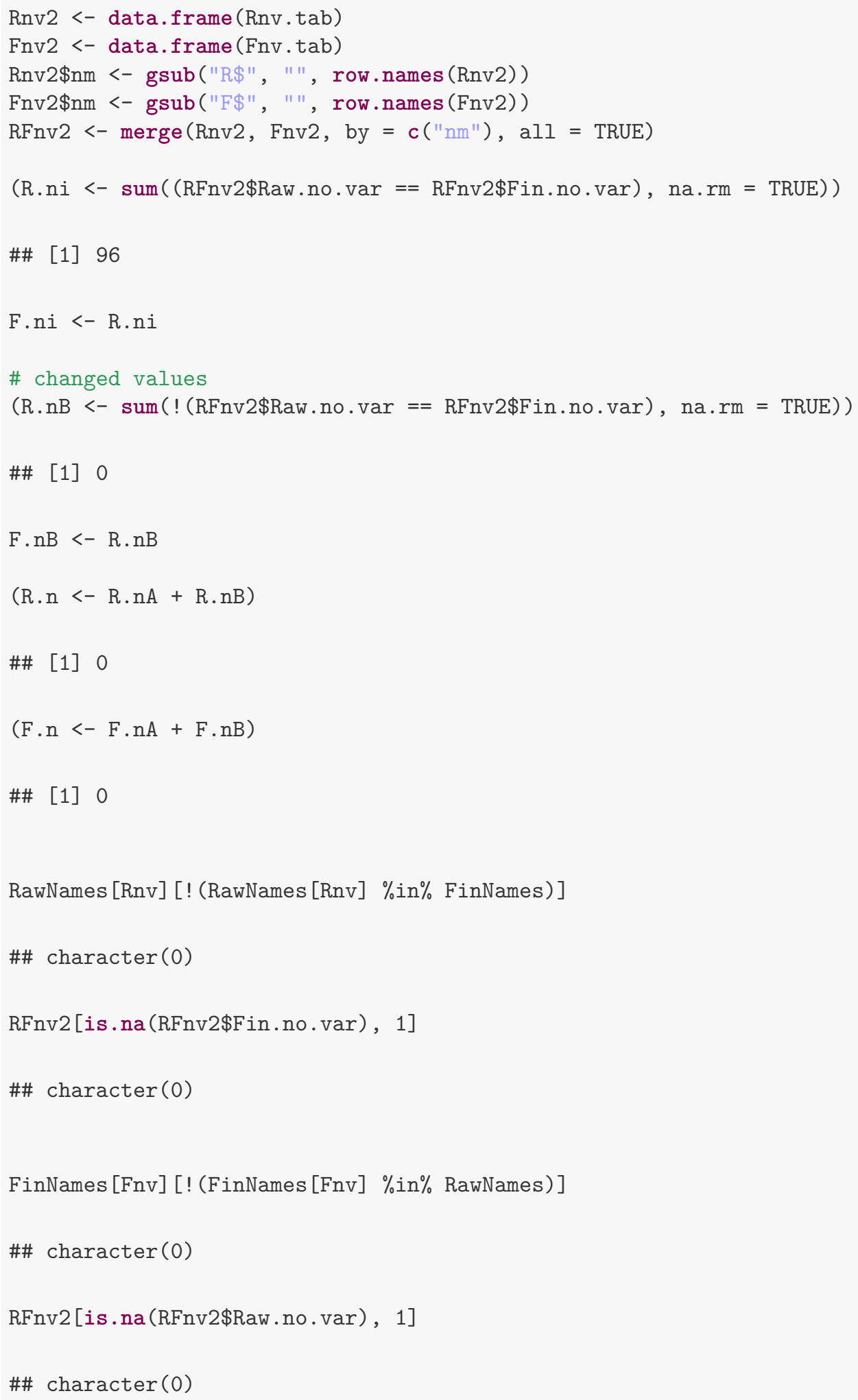

Release 2.0 96 variables without variation are identical in both data sets.

Release 3.0 96 variables without variation are identical in both data sets. 


\section{D.2. variation, but no bijective mapping}

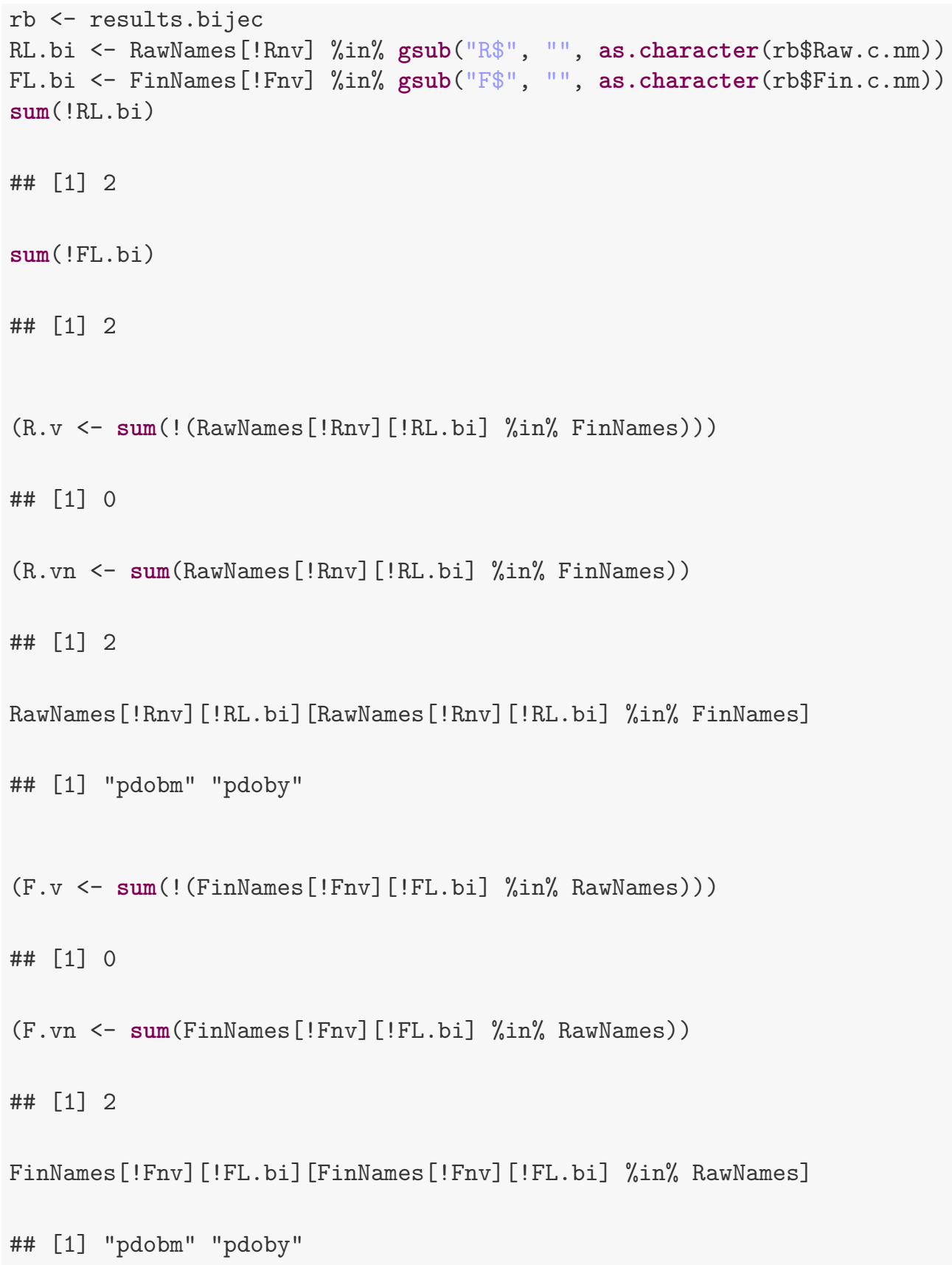

Release 2.0 2 variables share the name with one of the variables in Release 3.0 at least.

Release 3.0 2 variables share the name with one of the variables in Release 2.0 at least.

We compare all variables pairs (with variation) which share the same name but are not connected with a bijective mapping.

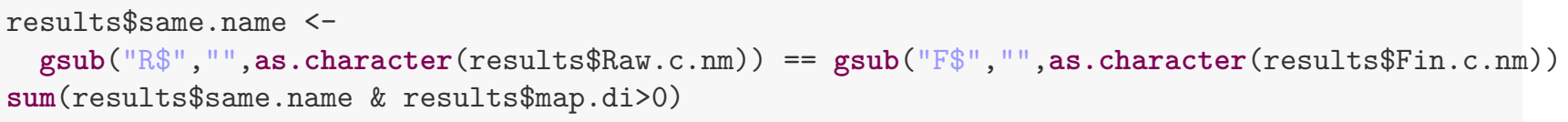


\#\# [1] 2

print(results [(results\$same.name \& results\$map.di>0),c(2,3,5,6,7,8)], row. names=FALSE)

\#\# Fin.c.nm Fin.c.ls Raw.c.nm Raw.c.ls map.di ed.di.sum

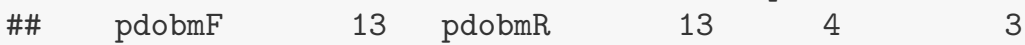

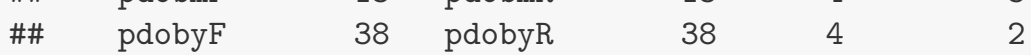

\section{D.3. variation and bijective mapping}

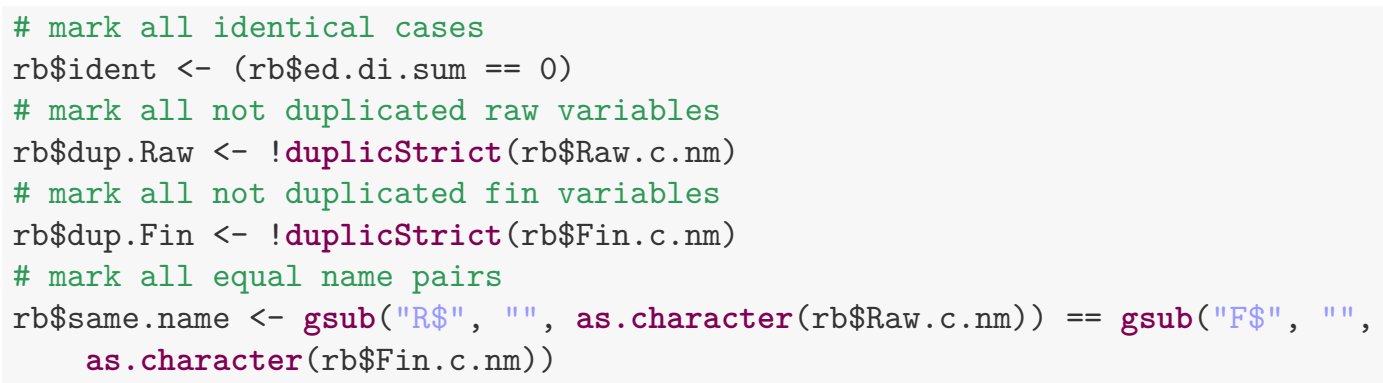




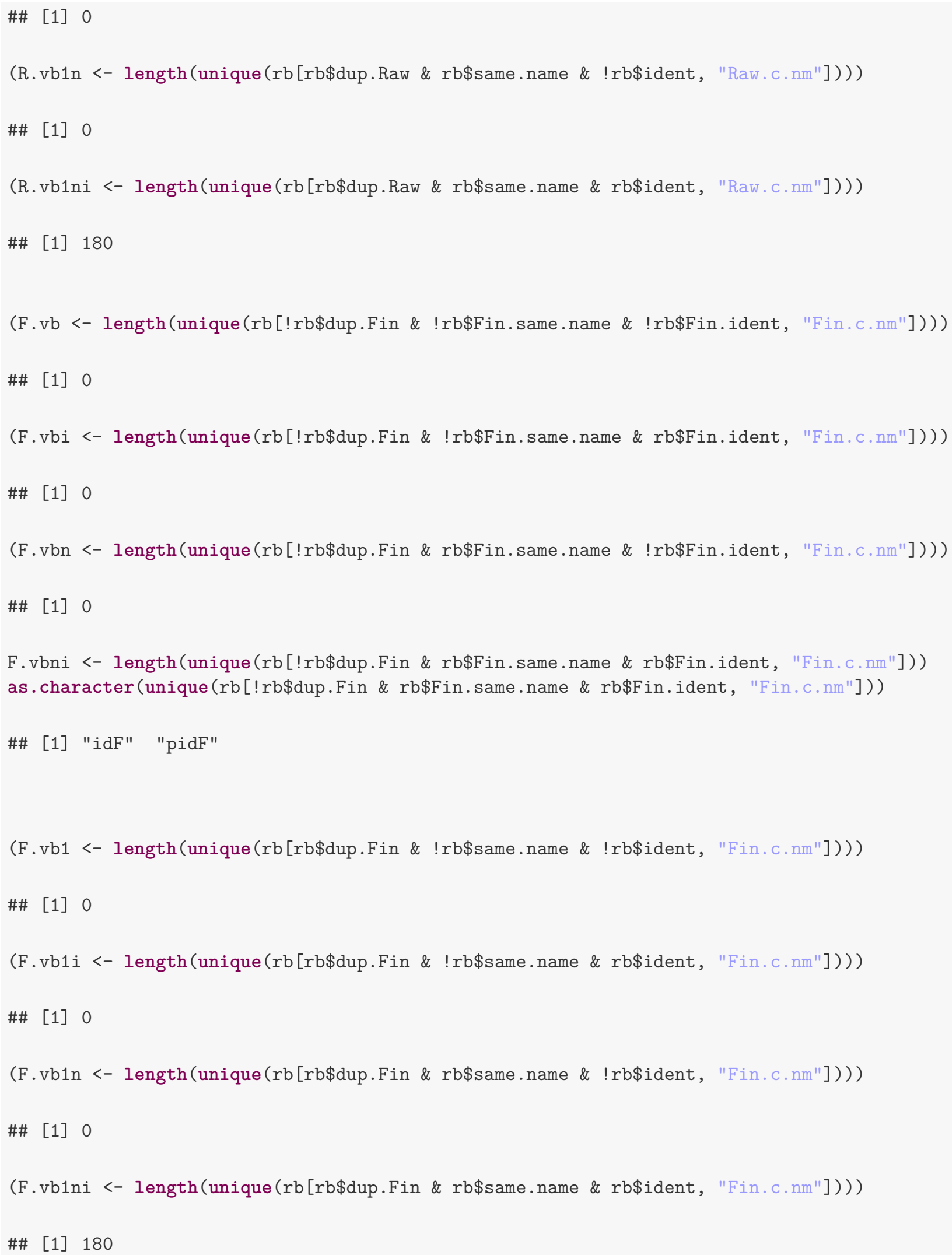




\section{D.4. comparison summary for partner2_DD.dta Release 2.0 and Release $\mathbf{3 . 0}$}

\begin{tabular}{rrr} 
class & Release 2 & Release 3 \\
\hline $\mathrm{x}$ & 0 & 0 \\
$\mathrm{n}$ & 0 & 0 \\
$\mathrm{ni}$ & 96 & 96 \\
\hline $\mathrm{v}$ & 0 & 0 \\
$\mathrm{vn}$ & 2 & 2 \\
\hline $\mathrm{vb}$ & 0 & 0 \\
$\mathrm{vbi}$ & 0 & 0 \\
$\mathrm{vbn}$ & 0 & 0 \\
vbni & 2 & 2 \\
$\mathrm{vb1}$ & 0 & 0 \\
$\mathrm{vb} 1 \mathrm{i}$ & 0 & 0 \\
$\mathrm{vb1n}$ & 0 & 0 \\
$\mathrm{vb} 1 \mathrm{ni}$ & 180 & 180 \\
\hline $\mathrm{sum}$ & 280 & 280
\end{tabular}

Most variables are unchanged (ni, vbni, vb1ni). A small group of variables (vn) had been changed.

\section{E. weights}

As usual we have first to load the produced data collection.

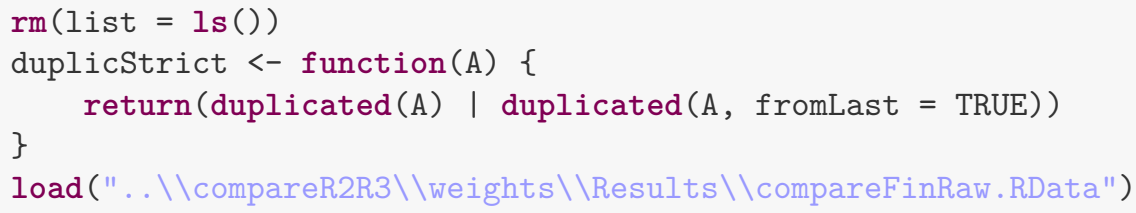

\section{E.1. no variation}

We compare the variables without variation first.

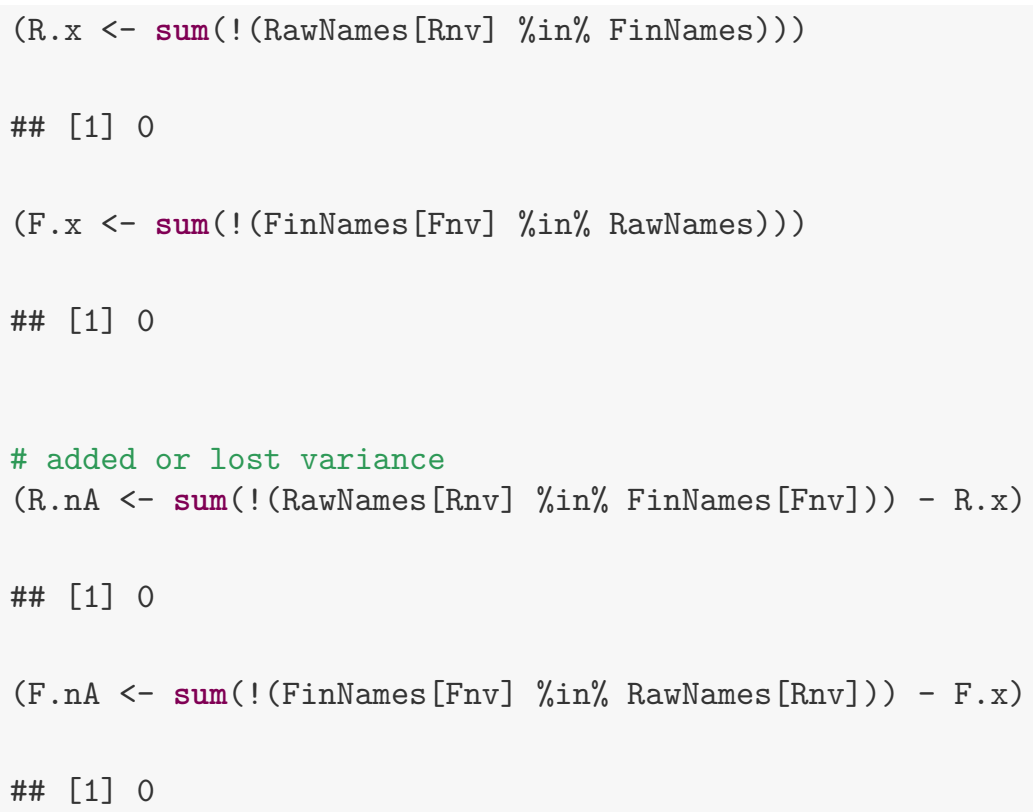




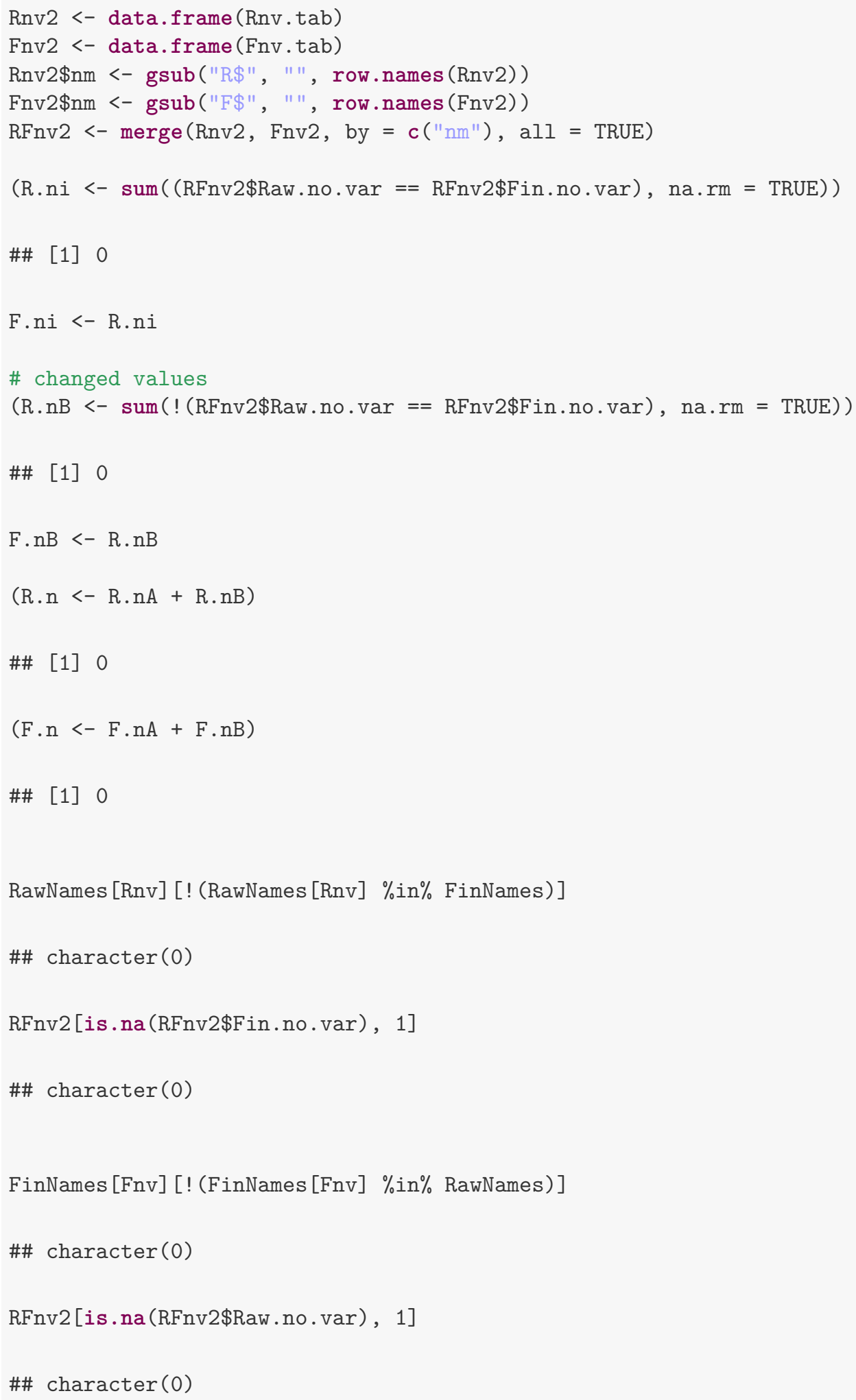

Release 2.0 No change for variables without variation.

Release 3.0 No change for variables without variation. 


\section{E.2. variation, but no bijective mapping}

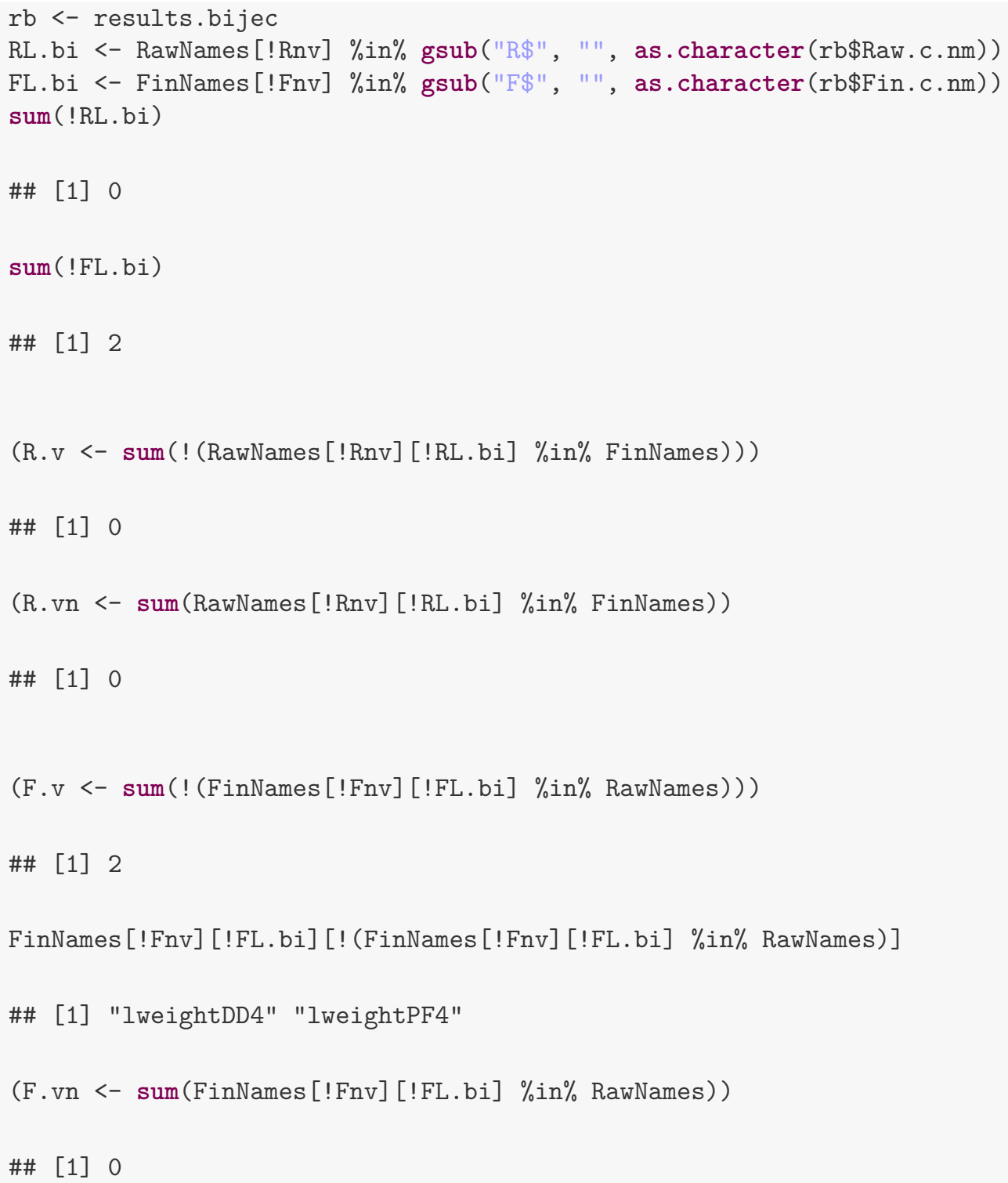

Release 2.0 No changed variables with variation but without any bijective mapping.

Release 3.0 For 2 variables with variation but without any bijective mapping exist no variables with the same name in the Release 2.0 data.

See the comparison table as an illustration.

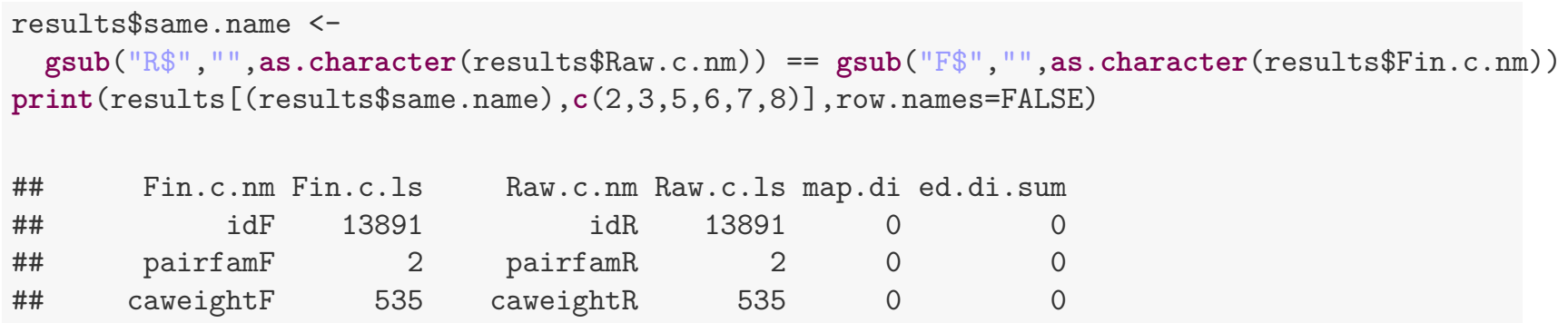




$\begin{array}{rrrrrrr}\# \# & \text { westF } & 2 & \text { westR } & 2 & 0 & 0 \\ \# \# & \text { ca1weightF } & 1470 & \text { ca1weightR } & 1470 & 0 & 0 \\ \# \# & \text { ddweightF } & 3 & \text { ddweightR } & 3 & 0 & 0 \\ \# \# & \text { ddcaweightF } & 581 & \text { ddcaweightR } & 581 & 0 & 0 \\ \# \# & \text { d1weightF } & 6 & \text { d1weightR } & 6 & 0 & 0 \\ \# \# & \text { d1ca1weightF } & 2380 & \text { d1ca1weightR } & 2380 & 0 & 0 \\ \# \# & \text { lweightDD3F } & 3 & \text { lweightDD3R } & 3 & 0 & 0 \\ \# \# & \text { lweightPF2F } & 7 & \text { lweightPF2R } & 7 & 0 & 0 \\ \# \# & \text { lweightPF3F } & 7 & \text { lweightPF3R } & 7 & 0 & 0\end{array}$

\section{E.3. variation and bijective mapping}

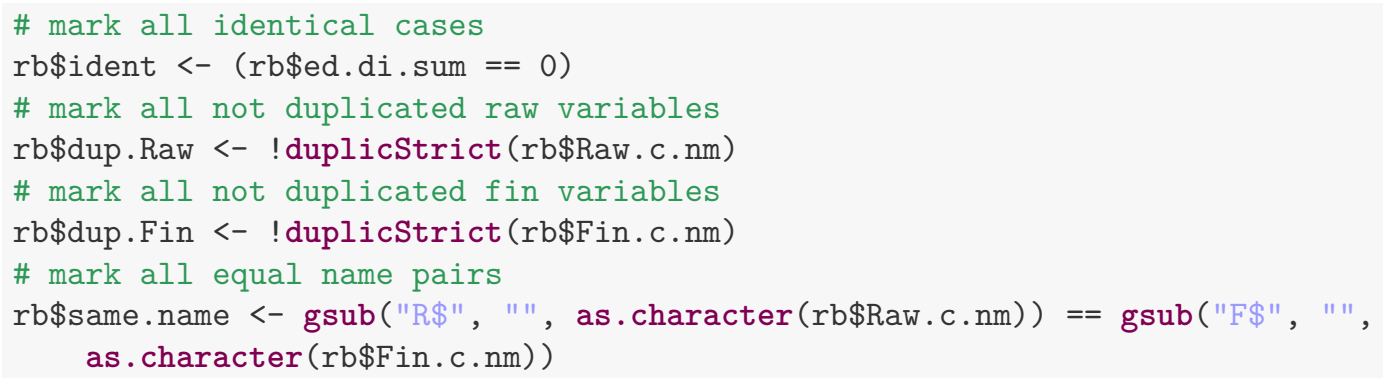


\#\# [1] 0

(R.vb1n <- length (unique(rb[rb\$dup.Raw \& rb\$same.name \& !rb\$ident, "Raw.c.nm"])))

\#\# [1] 0

R.vb1ni <- length (unique(rb[rb\$dup.Raw \& rb\$same.name \& rb\$ident, "Raw.c.nm"]))

as.character (unique(rb[rb\$dup. Raw \& rb\$same.name \& rb\$ident, "Raw.c.nm"]))

\#\# [1] "idR" "pairfamR" "caweightR" "westR"

\#\# [5] "ca1weightR" "ddweightR" "ddcaweightR" "d1weightR"

\#\# [9] "d1ca1weightR" "lweightDD3R" "lweightPF2R" "lweightPF3R"

(F.vb <- length(unique(rb[!rb\$dup.Fin \& !rb\$Fin.same.name \& !rb\$Fin.ident, "Fin.c.nm"])))

\#\# [1] 0

(F.vbi <- length(unique(rb[!rb\$dup.Fin \& !rb\$Fin.same.name \& rb\$Fin.ident, "Fin.c.nm"])))

\#\# [1] 0

(F.vbn <- length(unique(rb[!rb\$dup.Fin \& rb\$Fin.same.name \& !rb\$Fin.ident, "Fin.c.nm"])))

\#\# [1] 0

(F.vbni <- length(unique(rb[!rb\$dup.Fin \& rb\$Fin.same.name \& rb\$Fin.ident, "Fin.c.nm"])))

\#\# [1] 0

(F.vb1 <- length (unique (rb[rb\$dup.Fin \& !rb\$same.name \& !rb\$ident, "Fin.c.nm"])))

\#\# [1] 0

(F.vb1i <- length(unique(rb[rb\$dup.Fin \& !rb\$same.name \& rb\$ident, "Fin.c.nm"])))

\#\# [1] 0

(F.vb1n <- length(unique(rb[rb\$dup.Fin \& rb\$same.name \& !rb\$ident, "Fin.c.nm"])))

\#\# [1] 0

F.vb1ni <- length(unique(rb[rb\$dup.Fin \& rb\$same.name \& rb\$ident, "Fin.c.nm"]))

as.character (unique(rb[rb\$dup.Fin \& rb\$same.name \& rb\$ident, "Fin.c.nm"]))

\#\# [1] "idF" "pairfamF" "caweightF" "westF"

\#\# [5] "ca1weightF" "ddweightF" "ddcaweightF" "d1weightF"

\#\# [9] "d1ca1weightF" "lweightDD3F" "lweightPF2F" "lweightPF3F" 


\section{E.4. comparison summary for weights_DD.dta Release 2.0 and Release 3.0}

\begin{tabular}{rrr} 
class & Release 2 & Release 3 \\
\hline $\mathrm{x}$ & 0 & 0 \\
$\mathrm{n}$ & 0 & 0 \\
$\mathrm{ni}$ & 0 & 0 \\
\hline $\mathrm{v}$ & 0 & 2 \\
$\mathrm{vn}$ & 0 & 0 \\
\hline $\mathrm{vb}$ & 0 & 0 \\
$\mathrm{vbi}$ & 0 & 0 \\
$\mathrm{vbn}$ & 0 & 0 \\
$\mathrm{vbni}$ & 0 & 0 \\
$\mathrm{vb1}$ & 0 & 0 \\
$\mathrm{vb} 1 \mathrm{i}$ & 0 & 0 \\
$\mathrm{vb1n}$ & 0 & 0 \\
$\mathrm{vb1ni}$ & 12 & 12 \\
\hline $\mathrm{sum}$ & 12 & 14
\end{tabular}

All old variables are unchanged (vb1ni). A small number (v) had been added.

\section{Literatur}

[DemoDiff 2.0] Kreyenfeld, Michaela; Goldstein, Joshua; Walke, Rainer; Trappe, Heike; Huinink, Johannes (2013): Demographic Differences in Life Course Dynamics in Eastern and Western Germany (DemoDiff). GESIS Datenarchiv, Köln. ZA5684 Datenfile Version 2.0.0, http://dx.doi.org/doi:10.4232/demodiff.5684.2.0.0

[DemoDiff 3.0] Kreyenfeld, Michaela; Goldstein, Joshua; Walke, Rainer; Trappe, Heike; Huinink, Johannes (2013): Demographic Differences in Life Course Dynamics in Eastern and Western Germany (DemoDiff). GESIS Data Archive, Cologne. ZA5684 Data file Version 3.0.0, http://dx.doi.org/doi:10.4232/demodiff.5684.3.0.0

[TR-2012-003] Walke, Rainer; Müller, Andreas (2012): compareFinRaw.r - an R program to measure the difference between datasets. MPIDR Technical Report TR-2012-003.

[R 2.15] R Core Team (2012). R: A language and environment for statistical computing. R Foundation for Statistical Computing, Vienna, Austria. ISBN 3-900051-07-0, URL http://www.Rproject.org/. 